\title{
SETS OF METRICAL HYPOTHESES FOR GEOMETRY*
}

BY

\section{ROBERT L. MOORE}

In this paper is given a set of assumptions, $C$, concerning point, order $\dagger$ and congruence, which, together with a certain set of order assumptions $O, \ddagger$ a continuity assumption $K, \ddagger$ and a very weak parallel assumption $P_{0}, \ddagger$ are sufficient for the establishment of ordinary Euclidean geometry. Each assumption of this set is independent of all the remaining ones. Moreover congruence is here an independent symbol in the sense that it would be impossible $\S$ to prove that if the order assumptions $O, K$ and $P_{0}$ are true, then there must exist a relation satisfying the congruence assumptions $C$ with reference to the points and order in terms of which $O, K$ and $P_{0}$ are stated. According to Veblen $\|$ congruence would not, however, be thus independent if $P_{0}$ were replaced even by his still comparatively weak parallel assumption XI.

If in this categorical set of assumptions there is substituted for $K$ the assumption that every segment has a middle point, ${ }^{* *}$ and $P_{0}$ is replaced by the somewhat stronger assumption $P_{2}, \dagger \dagger$ then follows $\sharp \ddagger$ a geometry $\S \S$ in which a theory of proportion holds and rigid motion is possible.

Other alternative sets of assumptions are discussed. Among these an interesting set is that obtained by substituting for $P_{0}$ the postulate $P_{2}$ and for $K$ a postulate $I_{2}$ which may be roughly stated as follows: "If $B$ is within, and $B$ ' without, any circle $\alpha$, then each semicircle with $B^{\prime} B$ as diameter must have a point in common with the circle $\alpha . "\|\|$ Each assumption in the set composed of $P_{2}, I_{2}$

\footnotetext{
* Presented to the Chicago section of the American Mathematical Society, in a somewhat different form, A pril 22, 1905. Received for publication December 26, 1907.

$\dagger$ lt would be impossible (see Theorem 1 of $\$ 12$ ) to formulate two sets of assumptions, one set, $C$, being stated in terms of point and congrnence alone, and the other, $O$, being stated in terms of point and order alone, such that from $O$ and $C$ would follow a geometry, Euclidean with respect to the undefined symbols in terms of which $O$ and $C$.are stated.

$\ddagger O, K$ aud $P_{0}$ are all stated in terms of point and order.

§C. $\$ 10$.

\|These Transactions, vol. 5 (1904), pp. 343-334.

II Loc. cit. p. 346.

** Cl. end of $\S 1$ and first part of $\$ 6$.

†† See $\$ 6$.

$\ddagger \ddagger$ Cf. Theorem 1 of $\$ 6$.

$\S$ A geometry which is a consequence of this set of assumptions is a consequence of HILRERT's Axiom-groups I-IV and conversely.

III Cf. §7.
} 
and the assumptions $C$ and $O$ is independent of the remaining ones, and moreover this set is sufficient for the establishment of a geometry in which not only is there a theory of proportion and rigid motion but also all ordinary rule and compasses constructions are possible.

In this paper "proof" is used to mean an indication of a demonstration. "Hence," " by," " therefore," etc., are intended as suggesting certain relations and not necessarily as describing exactly the logical dependence of one statement upon another.

I wish to thank Professor E. H. Moone and Professor O. Veblen for suggestions and criticisms. Professor VeBLEN, who suggested the undertaking of this investigation, has not only made numerous suggestions and criticisms, but has given me much help in the way of actual collaboration.

\section{Assumptions for Euclidean geometry.}

O. VeBLeN's order " axioms" I and III-X.*

$C$. Assumptions concerning point and congruence, sometimes with reference to order, as follows :

$C_{\mathrm{1}_{a}}$. If $B \dagger$ is different from $C$ and $A^{\prime}$ is different from $B^{\prime}$ then there exists $a$ point $C^{\prime}$ such that $A^{\prime} B^{\prime} C^{\prime} \ddagger$ and $B C \equiv B^{\prime} C^{\prime}$.

$C_{1_{b}}$. If $B$ is different from $C$ and $A^{\prime}$ is different from $B^{\prime}$ then there is not more than one point $C^{\prime}$ such that $A^{\prime} B^{\prime} C^{\prime}$ and $B C \equiv B^{\prime} C^{\prime}$.

$C_{2}$. If $A$ is different from $B, A^{\prime}$ is different from $B^{\prime}, A^{\prime \prime}$ is different from $B^{\prime \prime}$, $A B \equiv A^{\prime} B^{\prime}$ and $A^{\prime} B^{\prime} \equiv A^{\prime \prime} B^{\prime \prime}$, then $A B \equiv A^{\prime \prime} B^{\prime \prime}$.

$C_{3}$. If $A B C, A^{\prime} B^{\prime} C^{\prime}, A B \equiv A^{\prime} B^{\prime}$ and $B C \equiv B^{\prime} C^{\prime}$, then $A C \equiv A^{\prime} C^{\prime}$.

$C_{4}$. If $A, B$ and $C$ are three non-collinear $\|$ points and $A^{\prime}, B^{\prime}$ and $C^{\prime}$ are three non-collinear points and $C A D, C^{\prime} A^{\prime} D^{\prime}, A B \equiv A^{\prime} B^{\prime}, B C \equiv B^{\prime} C^{\prime}, C A \equiv C^{\prime} A^{\prime}$, $C D \equiv C^{\prime} D^{\prime}$, then $B D \equiv B^{\prime} D^{\prime}$.

$K$. For the continuity assumption $K$ may be taken either VEBLEN's XI or a DEDEKIND cut assumption stated for the points of a single segment as follows : If there exists any segment then there exists some segment $A B$ such that if it is

* Loc. cit., pp. 344-346. From assumption $C_{1_{a}}$ below it follows that if $A$ and $B$ are two different points there is a point $C$ different from $B$ such that $A B C$. Veblesw's $A$ xiom II may be seen to be a consequence of this proposition and his Axioms I, III-VIII (of. a paper presented by me to the A merican Mathematical Society on October 26, 1907, but not yet published). Thus Axiom II is redundant in the set composed of VEBLEN's Axioms I-X together with my Axioms $C$.

† Capital letters are used to designate points.

$\ddagger \triangle B C$ used as a sentence means $A, B$ and $C$ are in the order $A B C$.

$\S B C$ as a simple word, unmodified by "line" or "ray," means the segment $B C$, i. e., the set of all points $X$ such that $B X C$.

\|Cf. VEBLEN, loc. cit., p. 345. Instead of "three non-collinear points" one might, in stating this assumption, use the phrase "three points such that neither $A B C, B C A$, nor $C A B$."

TCf. Vhron rise, Grundzüge der Geometrie, pp. 254-260. $C_{4}$ and $C_{3}$ might be stated together in one assumption. 
composed of two sets of points $[M]^{*}$ and $[N]$, each. set containing at least two points and no point $X$ of either set being either the same as $Y_{1}$ or such that $Y_{1} X Y_{2}$ where $Y_{1}$ and $Y_{2}$ are points of the other set, then there exists a point $C$ such that $M C N$ for every $M$ and $N$ different from $C$.

With the help of $O$, from either of these assumptions can be deduced a theorem to the effect that this DEDEkind cut proposition must apply to every segment as well as to the segment $A B$ (cf. VebLeN, loc. cit., pages 368 and 369).

$P_{0}$. If there exists any straight line and $a$ point not on it then there exists some straight line $a$, and sone point $A$, not on it, such that if $a$ and $A$ lie in a plane $\beta$ then in the plane $\beta$ there is not more than one straight line passing through $A$ and having no point in comnon with $a$.

\section{$\S 1$. Consequences of $O$ and $C$.}

On the basis of $O$ and $C$ we have the following propositions :

Theorem 1. If $A^{\prime}$ is a point and $A B$ is a segment, then on each ray $\ddagger$ starting from $A^{\prime}$ there is one and only one point $B^{\prime}$ such that $A B=A^{\prime} B^{\prime}$.

Proof. See $C_{1_{a}}$ and $C_{1_{b}}$.

Theorem 2. If $A B C$ and $C^{\prime}$ is on ray $A^{\prime} B^{\prime}$ and $A B \equiv A^{\prime} B^{\prime}$ and $A C \equiv A^{\prime} C^{\prime}$, then $A^{\prime} B^{\prime} C^{\prime}$.

Proof. There is, according to $C_{1_{\varepsilon}}$, a point $C^{\prime \prime}$ such that $A^{\prime} B^{\prime} C^{\prime \prime}$ and $B C \equiv B^{\prime} C^{\prime \prime}$. Hence, by $C_{3}, A C \equiv A^{\prime} C^{\prime \prime}$. But, according to hypothesis, $A C \equiv A^{\prime} C^{\prime}$. Therefore, by $C_{\mathrm{s}_{b}}, C^{\prime \prime}$ is $C^{\prime}$. But $A^{\prime} B^{\prime} C^{\prime \prime}$.

Corollary. If $B$ is on ray $A C$ and $B^{\prime}$ is on ray $A^{\prime} C^{\prime \prime}$ and $A B \equiv A^{\prime} B^{\prime}$ and $A C \equiv A^{\prime} C^{\prime}$, then $B C \equiv B^{\prime} C^{\prime}$.

Theorem 3.ई If $A$ is distinct from $B$, then $A B \equiv A B$.

Proof. According to Theorem 1 there exists, on ray $A B$, a point $B^{\prime}$ such that $A B \equiv A B^{\prime}$. Again there exists, on ray $A B$, a point $B^{\prime \prime}$ such that $A B^{\prime} \equiv A B^{\prime \prime}$. But, then, according to $C_{2}, A B \equiv A B^{\prime \prime}$ and thus (see Theorem 1) $B^{\prime}$ is $B^{\prime \prime}$. Hence $A B^{\prime} \equiv A B^{\prime}$. Therefore $A B \equiv A B^{\prime}$ and also $A B^{\prime} \equiv A B^{\prime}$. But if $B$ is not $B^{\prime}$ then either $A B B^{\prime}$ or $A B^{\prime} B$ and thus, by Theorem 2, $A B^{\prime} B^{\prime}$, which is impossible. So $B$ is $B^{\prime}$. But $A B \equiv A B^{\prime}$. Hence $A B \equiv A B$.

Theorem 4. If $A B \equiv A^{\prime} B^{\prime}$, then $A^{\prime} B^{\prime} \equiv A B$.

Proof. According to Theorem 1 there exists, on ray $A B$, a point $B^{\prime \prime}$ such that $A^{\prime} B \equiv A B^{\prime \prime}$. So, by $C_{2}$, Theorem 3 and Theorem $1, B^{\prime \prime}$ must be $B$. But $A^{\prime} B^{\prime} \equiv A B^{\prime \prime}$. Thus $A^{\prime} B^{\prime} \equiv A B$.

* The notation $[M]$ is used to denote a cet of elements any one of whioh may be called $M$.

†CR. Vebles, loo. cit., p. 345.

IIf $A$ and $B$ are two distinot points, then the set of all points [C] such that either $C$ is $B$, or $\triangle C B$, or $\triangle B C$, is called a ray or half line starting from $\triangle$. Such a ray is called the ray $A B$.

\& Ct. Hilberat' IV, 1 (soe page 12 of Hinberats The Foundations of Geometry, Townsend's translation). 
Theorem 5.* If $A B \equiv A^{\prime} B^{\prime}$ and $A B \equiv A^{\prime \prime} B^{\prime \prime}$, then $A^{\prime} B^{\prime} \equiv A^{\prime \prime} B^{\prime \prime}$.

Proof. This theorem is a logical consequence of Theorem 4 and $C_{2}$.

Theorem 6. If $A B C$ and $A C \equiv A^{\prime} C^{\prime}$, then there exists one and only one point $B^{\prime}$ such that $A^{\prime} B^{\prime} C^{\prime}$ and $A B \equiv A^{\prime} B^{\prime}$ and $B C \equiv B^{\prime} C^{\prime}$.

Proof. According to Theorem 1 there is one and only one point $B^{\prime}$ on ray $A^{\prime} C^{\prime}$ such that $A B \equiv A^{\prime} B^{\prime}$. According to Theorem 2 and its corollary, $A^{\prime} B^{\prime} C^{\prime}$ and $B C \equiv B^{\prime} C^{\prime}$.

Definition 1. $A^{\prime} B^{\prime}<A B$ means : there exists a point $P$ such that $A P B$ and $A^{\prime} B^{\prime} \equiv A P . \quad A^{\prime} B^{\prime}>A B$ means $A B<A^{\prime} B^{\prime}$.

Theorem 7. If $A B>A^{\prime} B^{\prime}, A B \equiv C D$ and $A^{\prime} B^{\prime} \equiv C^{\prime} D^{\prime}$, then $C D>C^{\prime} D^{\prime}$.

Proof. According to definition 1 there exists a point $P$ such that $A P B$ and $A^{\prime} B^{\prime} \equiv A P$. So, according to Theorem 6 , there exists $P^{\prime}$ such that $C P^{\prime} D$ and $A P \equiv C P^{\prime}$. By $C_{2}$ it follows that $A^{\prime} B^{\prime} \equiv C P^{\prime}$. Therefore, by hypothesis and Theorem 5, $C^{\prime} D^{\prime} \equiv C P^{\prime}$. So $C D>C^{\prime} D^{\prime}$, according to Definition 1 .

Theorem 8. If $A$ is different from $B$ and $A^{\prime}$ is different from $B^{\prime}$, then, of the three statements $A B \equiv A^{\prime} B^{\prime}, A B>A^{\prime} B^{\prime}, A B<A^{\prime} B^{\prime}$, one and only one is true.

Theorem 9. If $A B>A^{\prime} B^{\prime}$ and $A^{\prime} B^{\prime}>A^{\prime \prime} B^{\prime \prime}$, then $A B>A^{\prime \prime} B^{\prime \prime}$.

Definition 2. $A B+C D$ means the segment $A E$, where $E$ is a point such that $A B E$ and $C D \equiv B E$. If $A B>C D$ then $A B-C D$ means the segment $A E$ where $E$ is a point such that $B E A$ and $C D \equiv B E$.

Theorem 10. If $A B \equiv A^{\prime} B^{\prime}, C D \equiv C^{\prime} D^{\prime}$, then $A B+C D \equiv A^{\prime} B^{\prime}+C^{\prime} D^{\prime}$. We have the following rules of combination: $A B+C D \equiv C D+A B$, $(A B+C D)+E F \equiv A B+(C D+E F), A B-C D \equiv E F$ if and only if $A B \equiv C D+E F$.

A relation (called congruence) between angles $\dagger$ is introduced by the following definition :

Definition 3. $\Varangle A B C \equiv \Varangle A^{\prime} B^{\prime} C^{\prime}$ means that there exist points $D, D^{\prime}$, $E, E^{\prime}$, on rays $B A, B^{\prime} A^{\prime}, B C, B^{\prime} C^{\prime}$ respectively, such that $B D \equiv B^{\prime} D^{\prime}$, $D E \equiv D^{\prime} E^{\prime}, E B \equiv E^{\prime} B^{\prime}$.

Theorem 11. If $\Varangle B A C \equiv \Varangle B^{\prime} A^{\prime} C^{\prime}, B A \equiv B^{\prime} A^{\prime}, C A \equiv C^{\prime} A^{\prime}$, then $B C \equiv B^{\prime} C^{\prime}$.

Proof. By hypothesis and Definition 3 there exist on rays $A B, A C, A^{\prime} B^{\prime}$, $A^{\prime} C^{\prime}$, points $D, E, D^{\prime}, E^{\prime}$ respectively such that $A D \equiv A^{\prime} D^{\prime}, D E \equiv D^{\prime} E^{\prime}$, $E A \equiv E^{\prime} A^{\prime}$. By Theorem 1 there exist points $F, G, F^{\prime}, G^{\prime}$, such that $B A F$, $C A G, B^{\prime} A^{\prime} F^{\prime}, C^{\prime} A^{\prime} G^{\prime}, A F \equiv A^{\prime} F^{\prime}, A G \equiv A^{\prime} G^{\prime}$. By hypotheses and $C_{4}$ it follows that $E F \equiv E^{\prime} F^{\prime}$ and therefore, by Theorem 2 and another application of $C_{4}$, it may be proved that $F G \equiv F^{\prime} G^{\prime}$. Treating $A, B, C, A^{\prime}, B^{\prime}, C^{\prime}$,

* See Hilbere's IV, 2, loo. cit., p. 12.

† An angle is a point together with two nou-collinear rays which start from that point.

$\ddagger$ Cf. Veronese, loo. cit., p. 257. 
with reference to $A, G, F, A^{\prime}, G^{\prime}, F^{\prime}$ as $A, G, F, A^{\prime}, G^{\prime}, F^{\prime}$ were treated with reference to $A, D, E, A^{\prime}, D^{\prime}, E^{\prime}$, one may prove that $B C \equiv B^{\prime} C^{\prime}$.

Theorem 12.* $\Varangle A B C \equiv \Varangle A B C$.

Proof. Use Definition 3 and Theorem 3.

Theorem 13. If $\Varangle A B C \equiv \Varangle A^{\prime} B^{\prime} C^{\prime}$, then $\Varangle A^{\prime} B^{\prime} C^{\prime} \equiv \Varangle A B C$.

Proof. Use Definition 3 and Theorem 4.

Theorem 14. $\dagger$ If $\Varangle A B C \equiv \Varangle A^{\prime} B^{\prime} C^{\prime}$ and $\Varangle A B C \equiv \Varangle A^{\prime \prime} B^{\prime \prime} C^{\prime \prime}$, then $\Varangle A^{\prime} B^{\prime} C^{\prime} \equiv \Varangle A^{\prime \prime} B^{\prime \prime} C^{\prime \prime}$.

Proof. Use Definition 3 and Theorems 11 and 5.

Definition 4. Two angles are supplementary to (supplements of) each other if and only if they have a common vertex and one common side and their other sides form two rays of the same straight line.

Two angles are vertical angles if and only if they have a common vertex and their four sides, together with this common vertex, make up two straight lines.

Theorem 15. If $\Varangle H A K \equiv \Varangle H^{\prime} A^{\prime} K^{\prime}$ then each angle that is supplementary to $\Varangle H A K \equiv$ each angle that is supplemèntary to $\Varangle H^{\prime} A^{\prime} K^{\prime}$, and the angle that is vertical to $\Varangle H A K \equiv$ the angle that is vertical to $\Varangle H^{\prime} A^{\prime} K^{\prime}$.

Proof. See Theorem 11 and proof thereof.

Corollary. Vertical angles are congruent to each other.

Definition 5. $\triangle A B C \equiv \triangle A^{\prime} B^{\prime} C^{\prime}$ means : $A B \equiv A^{\prime} B^{\prime}, B C \equiv B^{\prime} C^{\prime}$, $C A \equiv C^{\prime} A^{\prime}, \Varangle A B C \equiv \Varangle A^{\prime} B^{\prime} C^{\prime}, \Varangle B C A \equiv \Varangle B^{\prime} C^{\prime} A^{\prime}, \Varangle C B A \equiv \Varangle C^{\prime} B^{\prime} A^{\prime}$.

Theorem 16. $\triangle A B C \equiv \triangle A^{\prime} B^{\prime} C^{\prime}$ if $A B \equiv A^{\prime} B^{\prime}, B C \equiv B^{\prime} C^{\prime}, C A \equiv C^{\prime} A^{\prime}$.

Proof: See Definitions 3 and 5.

Theorem 17.ई $\triangle A B C \equiv \triangle A^{\prime} B^{\prime} C^{\prime}$ if $A B \equiv A^{\prime} B^{\prime}, B C \equiv B^{\prime} C^{\prime}$, and $\Varangle A B C \equiv \Varangle A^{\prime} B^{\prime} C^{\prime}$.

Proof. See Theorem 11 and Definitions 3 and 5.

Theorem 18. If $\Varangle A B C \equiv \Varangle A^{\prime} B^{\prime} C^{\prime}$ and $D$ is in $\| \Varangle A B C$, then there exists a point $D^{\prime}$ in $\Varangle A^{\prime} B^{\prime} C^{\prime}$ such that $\Varangle A B D \equiv \Varangle A^{\prime} B^{\prime} D^{\prime}$ and $\Varangle D B C \equiv \Varangle D^{\prime} B^{\prime} C^{\prime}$.

Definition 6. $\Varangle B^{\prime} A^{\prime} C^{\prime}<\Varangle B A C$ means : there is a point, $P$, in $\Varangle B A C$ such that $\Varangle B^{\prime} A^{\prime} C^{\prime} \equiv \Varangle B A P . \Varangle B^{\prime} A^{\prime} C^{\prime}>\Varangle B A C$ means : $\Varangle B A C<\Varangle B^{\prime} A^{\prime} C^{\prime}$.

Theorem 19. If $\Varangle B A C>\Varangle B^{\prime} A^{\prime} C^{\prime}, \Varangle B A C \equiv \Varangle D E F, \Varangle B^{\prime} A^{\prime} C^{\prime} \equiv \Varangle D^{\prime} E^{\prime} F^{\prime}$, then $\Varangle D E F>\Varangle D^{\prime} E^{\prime} F^{\prime \prime}$.

Theorem 20. If $\Varangle B A C>\Varangle B^{\prime} A^{\prime} C^{\prime}$ and $\Varangle B^{\prime} A^{\prime} C^{\prime}>\Varangle B^{\prime \prime} A^{\prime \prime} C^{\prime \prime}$, then $\Varangle B A C>\Varangle B^{\prime \prime} A^{\prime \prime} C^{\prime \prime}$.

Theorem 21. If $A B C$ is a $\triangle$ and $A C \equiv B C$, then $\Varangle A B C \equiv \Varangle A C B$.

* See Hilbert's IV, 4, loc. oit., page 14.

† See Hilbert's IV, 5, lov. cit., page 14. $\triangle A B C$ (triangle $A B C$ ) means the three noncollinear points $A, B$, and $C$.

† See VRRONRSE; loc. cit., page 254.

\& See HILBERT's, IV, 6, loc. cit., page 15.

$\|$ If $A B C$ is an angle, the two rays, $B A$ and $B C$, together with the vertex $B$, divide the plane $\triangle B C$ into two regions. That one of these regions which contains points $P$ such that $A P C$ is called the interior of $\Varangle A B C$. Cf. VEBLEN, loc. cit., pp. 363-365. 
Proof. Use Theorem 3 and Definition 3.

Definition 7. "A middle point of the segment $A B$ " means a point $M$, on the straight line $A B$, such that $A M \equiv M B$.

Theorem 22. If $M$ is a middle point of $A B$, then $A M B$.

Proof. Use Definition 7 and $C_{1_{b}}$.

Theorem 23. No segment has two middle points.

Proof. Use Definition 1 and Theorems 22, 7 and 8.

I do not know as yet whether from $O$ and $C$ it follows that every segment has one middle point. This proposition (statement) that every segment has a middle point will be denoted by the symbol $M$.

\section{$\S 2$. Two consequences of $O, C$ and $M$.}

In $\S 1$ a number of propositions were deduced as consequences of $O$ and $C$. In the present paragraph it will be shown that, if $M$ is assumed in addition to $O$ and $C$, then follow two propositions concerning angles.

The symbol $A_{1}$ will be used to denote the proposition : If $A B C, A B \equiv B C$, and $D$ is not on the straight line $A B$, then there is, in the half-plane $D-A B,{ }^{*} a$ point $E$ such that $A D \equiv B E$ and $B D \equiv C E$.

The symbol $A_{-2}$ denotes the proposition: If $C$ is not straight line $A B$, then, in the half-plane $C-A B$, there is no point $C^{\prime}$, different from $C$, such that $A C \equiv A C^{\prime}$ and $B C \equiv B C^{\prime} .+$

Theorem 1. From $O, C$ and $M$ follows $A_{-2}$.

Proof. If this theorem is not true, then there exists a space for which $O, C$ and $M$ are true but which contains four distinct coplanar points $A, B, C^{\prime}, C^{\prime \prime}$ such that $C^{\prime}$ and $C^{\prime \prime}$ are on the same side of $A B$ and moreover $E C^{\prime} \equiv B C^{\prime \prime}$ and $C^{\prime} A \equiv C^{\prime \prime} A$. In case the straight lines $C^{\prime} C^{\prime \prime}$ and $A B$ have a oint $P$ in common, then with use of Theorems 15, 16, 17 and 3 it may be seen that $\triangle A P C^{\prime} \equiv \triangle A P C^{\prime \prime}$, and thus $P C^{\prime} \equiv P C^{\prime \prime}$, but this is impossible according to $C_{1_{b}}$ and the hypothesis that $C^{\prime}$ and $C^{\prime \prime}$ are on the same side of $A B$. In case the straight lines $C^{\prime} C^{\prime \prime}$ and $A B$ have no point in common, then $A$ and $B$ are on the same side of the straight line $C^{\prime} C^{\prime \prime}$. According to $M$ and Theorem 22 , there is a point $S$ such that $C^{\prime \prime} S C^{\prime}$ and $C^{\prime \prime} S=S C^{\prime}$. There exists, as may be seen by use of $O$, a point $P$ such that $P S A$ and segment $P B$ has a point $S^{\prime \prime}$ in common with segment $C^{\prime} C^{\prime \prime}$. Consider $\triangle$ 's $A S C^{\prime}$ and $A S C^{\prime \prime}$. $A S \equiv A S$ by Theorem 3 , while, by hypothesis, $A C^{\prime} \equiv A C^{\prime \prime}$ and $C^{\prime} S \equiv S C^{\prime \prime}$. Hence, by Theorem 16, $\triangle A S C^{\prime} \equiv \triangle A S C^{\prime \prime}$. With use of $C_{2}, C_{4}$ and The-

* By a hall-plane is meant one of the two regions into which a plane is decomposed by a straight line which lies in it (ot. VeBLEN, loc. cit., pp. 363-365). If the straight line $A B$ thus decumposes a plane into two regions and $D$ lies in one of these regions then this region is called the hall-plane $D-A B$.

†Thus, according to Definition $3, \Varangle B A D \equiv C B E$ and $A_{1}$ is, then, a weak form of the proposition given in the statement of Theorem 4 of $\$ 5$. Cf. HrLBrar's IV, 4, loo. cit., p. 14.

fCR. HILbert's IV, 4, loo. oit., p. 14. 
orem 3 it follows that $P C^{\prime \prime} \equiv P C^{\prime}$. Hence, by hypothesis, Theorem 3 and Definition 3, it follows that $\Varangle C^{\prime \prime} B S^{\prime} \equiv \Varangle C^{\prime} B S^{\prime}$ and, consequently, by means of Theorems 17 and 3 and Definition 5 , that $C^{\prime \prime} S^{\prime} \equiv S^{\prime} C^{\prime}$, but this is impossible according to theorem 23.

Corollary.* Given an angle $B A C$ and a ray $A^{\prime} B^{\prime}$, there is, in a given plane containing $A^{\prime} B^{\prime}$ and on a given side of $A^{\prime} B^{\prime}$, not more than one ray $A^{\prime} C^{\prime}$ such that $\Varangle B A C \equiv \Varangle B^{\prime} A^{\prime} C^{\prime}$.

Theorem 2. From $O, C$ and $M$ follows $A_{1}$.

Proof. Suppose $A B C, A B \equiv B C$ and $D$ is not on the straight line $A C$. According to $M$ and Theorem 22 , there is a point $S$ such that $A S B$ and $A S \equiv S B$. By $C_{1 a}$ there exists a point $E^{\prime}$ such that $D S E^{\prime}$ and $D S \equiv S E^{\prime}$. According to Theorem 17 and Corollary to theorem $15, \Varangle S A D \equiv \Varangle S B E^{\prime}$. But by $C_{1_{a}}$ there is a point $E$ such that $E^{\prime} B E$ and $A D \equiv B E$. By theorems 13 and 14 and Corollary to Theorem 15, $\Varangle D A S \equiv \Varangle C B E$. But $A B \equiv B C$ and $A D \equiv B E$. Hence, by theorem $11, B D \equiv C E$.

\section{§3. Consequences of $\mathrm{O}, \mathrm{C}$ and $\mathrm{A}_{-2}$.}

In $\S 2$ it has been shown that if $M$ is assumed in addition to $O$ and $C$, then there follow the two propositions $A_{1}$ and $A_{-2}$. On the basis of $O, C$ and $A_{-2}$ we have the following propositions.

Theorem 1. $\triangle A B C \equiv \triangle A^{\prime} B^{\prime} C^{\prime}$ if $\Varangle A B C \equiv \Varangle A^{\prime} B^{\prime} C^{\prime}, \Varangle B A C \equiv \Varangle B^{\prime} A^{\prime} C^{\prime}$ and $A B \equiv A^{\prime} B^{\prime}$.

Proof. According to Theorem 1 of $\S 1$ there is a point $C^{\prime \prime}$ on ray $A^{\prime} C^{\prime}$ such that $A C \equiv A^{\prime} C^{\prime \prime}$. According to hypothesis and Theorem 17, $\triangle A B C \equiv \triangle A^{\prime} B^{\prime} C^{\prime \prime}$ and thus (see Definition 5) $\Varangle A B C \equiv \Varangle A^{\prime} B^{\prime} C^{\prime}$. But $\Varangle A B C=\Varangle A^{\prime} B^{\prime} C^{\prime}$. By $A_{-2}$ and Theorem 1 of $\S 1$ it follows that either $C^{\prime \prime}$ is $C^{\prime}$ or, if not, then $B^{\prime}$ is on the straight line $C^{\prime \prime} C^{\prime}$ and, thus, on the straight line $A^{\prime} C^{\prime}$, contrary to hypothesis. So $C^{\prime \prime}$ is $C^{\prime}$. But $\triangle A B C \equiv$ $\triangle A^{\prime} B^{\prime} C^{\prime \prime}$. Hence $\triangle A B C \equiv A^{\prime} B^{\prime} C^{\prime}$.

Theorem 2. $\dagger$ Of the three statements, $\Varangle B A C<\Varangle B^{\prime} A^{\prime} C, \Varangle B A C \equiv \Varangle B^{\prime} A^{\prime} C^{\prime}$, $\Varangle B A C>\Varangle B^{\prime} A^{\prime} C^{\prime}$, not more than one is true.

Proof. Make use of Definition 5 and $A_{-2}$. For instance suppose $\Varangle B A C>\Varangle B^{\prime} A^{\prime} C^{\prime}$. Then, according to Definition 6 , there exists a point $D$, in $\Varangle B A C$, such that $\Varangle B^{\prime} A^{\prime} C^{\prime} \equiv \Varangle B A D$. So, if $\Varangle B A C \equiv \Varangle B^{\prime} A^{\prime} C^{\prime}$, then $\Varangle B A C \equiv \Varangle B A D$, and this could not be, in view of $A_{-2}$; while, if $\Varangle B A C<\Varangle B^{\prime} A^{\prime} C^{\prime}$, then there exists, in $\Varangle B^{\prime} A^{\prime} C^{\prime}$, a point $D^{\prime}$ such that $\Varangle B A C \equiv \Varangle B^{\prime} A^{\prime} D^{\prime}$. Then, according to original hypothesis and Theorem $19, \Varangle B^{\prime} A^{\prime} D^{\prime}>\Varangle B^{\prime} A^{\prime} C^{\prime}$. Hence there exists, in $\Varangle B^{\prime} A^{\prime} D^{\prime}$, and at the same

"See HILBERT's IV, 4, loo. oit., p. 14.

t Ohserve that it is not here stated that one of these statements is true. See Corollary to Theorem 2 of $\$ 4$. 
time in $\Varangle B^{\prime} A^{\prime} C^{\prime}$, a point $G^{\prime \prime}$ such that $\Varangle B^{\prime} A^{\prime} C^{\prime} \equiv \Varangle B^{\prime} A^{\prime} G^{\prime \prime}$, but this could not be according to $A_{-2}$. So, if $\Varangle B A C>\Varangle B^{\prime} A^{\prime} C^{\prime}$, then neither $\Varangle B A C<\Varangle B^{\prime} A^{\prime} C^{\prime}$ nor $\Varangle B A C \equiv \Varangle B^{\prime} A^{\prime} C^{\prime}$.

Theorem 3. If $H G F$, and $I$ is not on the straight line $H F$ and $A$ is different from $C$, and $B, D, A$ and $C$ are coplanar but neither $B$ nor $D$ is on the straight line $A C$, and $\Varangle B A C \geqq \Varangle F G I$ and $\Varangle A C D \geqq \Varangle I G H$, then the rays $C D$ and $A B$ do not meet.

Proof. Of course, the rays $A B$ and $C D$ cannot meet unless they are on the same side of the straight line $A C$. Suppose they are on the same side of $A C$. According to hypothesis and Definition 6, there exist points $P$ and $S$ such that either ray $C S$ is ray $C D$ or ray $C S$ is in $\Varangle D C A$ and moreover either ray $A P$ is ray $A B$ or ray $A P$ is in $\Varangle C A B$ and furthermore $\Varangle A C S \equiv \Varangle H G I$ and $\Varangle C A P \equiv \Varangle F G I$. Evidentiy, then, rays $C D$ and $A B$ cannot meet unless rays $C S$ and $A P$ meet. Suppose rays $C S$ and $A P$ do meet in a point $M$. There exists, according to $C_{1_{a}}$, a point $M^{\prime}$ such that $M C M^{\prime}$ and $A M \equiv C M^{\prime}$. Now, by Theorems 15,13 and $14, \Varangle C A M \equiv \Varangle A C M^{\prime}$. Hence, by Theorems 3 and 17 and Definition $5, \Varangle C A M^{\prime} \equiv \Varangle A C M$. But, by Theorems 15,13 and $14, \Varangle A C M \equiv \Varangle P^{\prime} A C$, where $P^{\prime}$ is any point such that $P^{\prime} A P$. It follows, by means of $A_{-2}$, that the points $M, A$ and $M^{\prime}$ are collinear. So the straight lines $A P$ and $C S$ unite in two* points $M$ and $M^{\prime}$, but this is impossible. Therefore rays $A P$ and $C S$ do not meet.

Corollary. If $H(x F$, and $I$ is not on the straight line $H F$, and $A, B$ and $C$ are three non-collinear points, and $D$ is in the half-plane $B-A C$, and $\Varangle B A C \equiv \Varangle F G I$ and $\Varangle A C D \equiv \Varangle I G H$, then the lines $C D$ and $A B$ have no point in common.

Theorem 4. If $A B C$ is a triangle and $B C>A C$, then $\Varangle A>\Varangle B$.

Proof. By hypotheses, Theorem 7, and Definition 1, there exists, between $B$ and $C$, a point $D$ such that $A C \equiv C D$. Since $D$ is between $B$ and $C$, it follows from Theorem 12 and Definition 6 that $\Varangle B A C>\Varangle D A C$. But, by construction and Theorem 21, $\supset A C \equiv \Varangle A D C$. Hence, by Theorems 12 and 19, $\measuredangle B A C>\Varangle C D A$. But, by Theorem 2 and Corollary 2 to Theorem 3 of $\S 3, \Varangle C D A>\Varangle A B C$. Hence, by Theorems 3 and 19 of $\S 1$, $\Varangle B A C>\Varangle A B C$.

Theorem 5. If $D$ is within $\Varangle B A C$ and $D^{\prime}$ is within $\Varangle B^{\prime} A^{\prime} C^{\prime}$ and $\Varangle B A D \equiv \Varangle B^{\prime} A^{\prime} D^{\prime}$ and $\Varangle D A C \equiv \Varangle D^{\prime} A^{\prime} C^{\prime}$, then $\Varangle B A C \equiv B^{\prime} A^{\prime} C^{\prime}$.

Proof. It may be easily seen that there exist points $E, F, E^{\prime}, G^{\prime \prime}$ on rays $A C, A D, A^{\prime} C^{\prime}, A^{\prime} D^{\prime}$ respectively such that $A E \equiv A^{\prime} E^{\prime}, A F \equiv A^{\prime} F^{\prime}$, and $E F$ and $E^{\prime} F^{\prime}$ cut rays $A B$ and $A^{\prime} B^{\prime}$ in two points $G$ and $G^{\prime}$ respectively. By hypothesis and Theorem $17, \Varangle A F E \equiv \Varangle A^{\prime} F^{\prime} E^{\prime}$ and $E F \equiv \Varangle E^{\prime} F^{\prime}$. But it is clear that $E F G$ and $E^{\prime} F^{\prime} G^{\prime}$. Hence, by Theorem 15,

\footnotetext{
* That $M$ and $M^{\prime}$ are distinct is a consequence of $O$.
} 
$\Varangle A F G \equiv \Varangle A^{\prime} F^{\prime} G^{\prime}$. But $\Varangle G A F \equiv \Varangle G^{\prime} A^{\prime} F^{\prime}$ and $A F \equiv A^{\prime} F^{\prime}$. Hence, by Theorem $1, F G \equiv F^{\prime} G^{\prime}$. But $E F \equiv E^{\prime} F^{\prime}$. Hence, by $C_{3}, E G \equiv E^{\prime} G^{\prime}$. But $A E \equiv A^{\prime} E^{\prime}$ and $A G \equiv A^{\prime} G^{\prime}$. Hence, by Definition $3, \Varangle B A C \equiv \nvdash^{\prime} B^{\prime} A^{\prime} C^{\prime}$.

Definition 7. A right angle is an angle that is congruent to its supplement. A straight line $a$ is perpendicular to a straight line $b$ if and only if they intersect in a point $O$ and there exist two points, $A$ and $B$, different from $O, A$ lying on $a$ and $B$ lying on $b$, such that $\Varangle A O B$ is a right angle.

Corollary. Every angle which is congruent to a right angle is itself a right angle. If the straight line $a$ is perpendicular to the straight line $b$, then $b$ is perpendicular to $a$ and if $A$ and $B$ are any two points different from $O, A$ lying on $a$ and $B$ lying on $b$, then $\Varangle A O B$ is a right angle.

Proof. See Theorems 12, 14 and 15 of $\S 1$.

Theorem 6. In each plane there is a right angle.

Proof. Suppose $A, B, C$, are three distinct non-collinear points of a given plane. According to Theorem 1 of $\S 1$ there exists, on ray $A C$, a point $B^{\prime}$ such that $A B \equiv A B^{\prime}$. There is a point $D$ such that $A D B$ and, according to Theorem 6 of $\S 1$, there is a point $D^{\prime}$ such that $B^{\prime} D^{\prime} A, B D \equiv B^{\prime} D^{\prime}$ and $D A \equiv D^{\prime} A$. According to $O$, there is a point $P$ such that $B P D^{\prime}$ and $B^{\prime} P D$. Now $\Varangle B^{\prime} B A \equiv \Varangle B B^{\prime} A$ (see Theorem 21). Hence, by Theorems 3 and 17 of $\S 1, \triangle B B^{\prime} D \equiv \triangle B^{\prime} B D^{\prime}$. Thus $\Varangle B^{\prime} D^{\prime} B \equiv \Varangle B^{\prime} D B$ and therefore, by Theorem 15, it follows that $\Varangle P D^{\prime} A \equiv \Varangle P D A$. Moreover, by Theorems 17 and 12 and Definition $5, \Varangle P B D \equiv \Varangle P B^{\prime} D^{\prime}$. Hence $\triangle B^{\prime} P D^{\prime} \equiv \triangle B P D$ according to Theorem 1. Thus $P D^{\prime} \equiv P D$. Now also $D A \equiv D^{\prime} A$. Hence, by Theorem 3 of $\S 1$ and Definition $3, \Varangle B^{\prime} A P \equiv \Varangle B A P$. According to $O$, there is a point $M$, on $A P$, such that $B^{\prime} M B$. By Theorems 17 and 3 and Definition $5, M B^{\prime} \equiv M B$ and $\Varangle A M B^{\prime} \equiv \Varangle A M B$. Thus $\Varangle A M B$ is a right angle (see Definition 7).

Corollary. If $B A C$ is an angle, then there exists, within $\Varangle B A C$, a point $O$ such that $\Varangle B A O \equiv \Varangle O A C$.

Theorem 7. If there is one perpendicular $p$ to a straight line $a$, then, through each point of $a$, and lying in the plane $p a$, there is one and only one straight line perpendicular to $a$.

Proof. Suppose $O$ is the point at which $p$ intersects $a, C$ is any other point of $a$, and $P$ is any other point of $p$. By $C_{1_{a}}$ there exists a point $P^{\prime}$ such that $P O P^{\prime}$ and $P O \equiv O P^{\prime}$. There is also a point $P^{\prime \prime}$ such that $P^{\prime} C P^{\prime \prime}$ and $P C \equiv C P^{\prime \prime}$. There is (see Corollary to Theorem 6) a ray $C M$ in $\Varangle P C P^{\prime \prime}$ such that $\Varangle P C M \equiv \Varangle M C P^{\prime \prime}$. Now, also, $\Varangle P C O \equiv \Varangle P^{\prime} C O \equiv \Varangle P^{\prime \prime} C O$, where $O C O^{\prime}$. Hence (see Theorem 5) $\Varangle M C O \equiv \Varangle M C O^{\prime}$. Hence, by definition $7, M C$ is perpendicular to $a$ at the point $C$.

Suppose there is, in the plane $p a$, another perpendicular to $a$ at the point $C$. Suppose $N$ is a point of this new perpendicular on the same side of $a$ as $M$ and 
suppose $M C M^{\prime}$ and $N C N^{\prime}$. Ray $C N$ must lie either in $\Varangle O C M$ or in $\Varangle O^{\prime} C M$. Suppose it lies in $\Varangle O C M$. Then ray $C N^{\prime}$ lies in $\Varangle O^{\prime} C M^{\prime}$. According to Definition 7, Theorem 12, and Theorem 15, $\Varangle M C O \equiv \Varangle M^{\prime} C O$ and $\Varangle N C O \equiv \Varangle N^{\prime} C O$. By Theorem 18, there is a point $N^{\prime \prime}$ in $\Varangle M^{\prime} C O$ such that $\Varangle O C N \equiv O C N^{\prime \prime}$. But $\Varangle O C N \equiv \Varangle O C N^{\prime}$ and ray $C N^{\prime}$ is in $\Varangle O^{\prime} C M^{\prime}$ and thus is different from $C N^{\prime \prime}$. But this is impossible according to $A_{-2}$. Thus $C N$ cannot be in $\Varangle O C M$. Similarly it cannot be in $\Varangle O C M$. There is, then, in the plane $p a$ and passing through $C$, not more than one straight line perpendicular to $a$.

Theorem 8. If there is one perpendicular $p$ to a straight line $a$, and $P$ is any point in the plane $p a$, then through $P$, and lying in this plane, there is one and only one perpendicular to $a$.

Proof. If $P$ is on $a$ cf. Theorem 7. If $P$ is not on $a$, suppose $F$ is a point of $p$ on the same side of $a$ as $P$ and $O$ is the point where $p$ meets $a$. If $P$ is on $O F$, then certainly, through $P$, there is a perpendicular $(O F)$ to $A B$. If $P$ is not on $O F$, it is on one side of it. Suppose $H$ is a point of $a$ that is on the same side of $O F$ as $P$. Then $P$ lies in $\Varangle H O F$. There is (by $C_{1_{a}}$ ) a point $F^{\prime}$ such that $F O F^{\prime}$ and $O F \equiv O F^{\prime}$. According to Theorem 18 there is, in $\Varangle H O F^{\prime}$, a point $P^{\prime}$ such that $\Varangle P O H \equiv \Varangle H O P^{\prime}$. On ray $O P^{\prime}$ there is a point $P^{\prime \prime}$ such that $O P \equiv O P^{\prime \prime}$. Since $P$ and $P^{\prime \prime}$ are on opposite sides of $O H$ and on the same side of $O F$, there is a point $M$ of ray $O H$ such that $P M P^{\prime \prime}$. According to Theorems 3 and 17 of $\S 1, \triangle O M P \equiv \triangle O M P^{\prime \prime}$. So $\Varangle P M O \equiv \Varangle P^{\prime \prime} M O$ and thus $P M$ is perpendicular to $a$.

Now suppose that through $P$ there is, in this same plane, another perpendicular to $a$. Suppose this new perpendicular meets $a$ in the point $M^{\prime}$. There is a point $p^{\prime \prime \prime}$ such that $P M^{\prime} P^{\prime \prime \prime}$. According to hypothesis, Definition 7 and Theorems 13 and 14, $\Varangle M M^{\prime} P \equiv \Varangle M M^{\prime} P^{\prime \prime \prime}$ and $\Varangle M^{\prime} M P \equiv \Varangle M^{\prime} M P^{\prime \prime}$. According to Theorem 17, $\triangle P M M^{\prime} \equiv \triangle P^{\prime \prime} M M^{\prime}$. So $\Varangle P M^{\prime} M \equiv \Varangle M M^{\prime} P^{\prime \prime}$. But $\Varangle P M^{\prime} M \equiv \Varangle P^{\prime \prime \prime} M^{\prime} M$. Hence, according to $A_{-2} ; P^{\prime \prime}$ must lie on ray $M^{\prime} P^{\prime \prime}$ and thus the straight lines $M P$ and $M^{\prime} P$ intersect in two points $P$ and $P^{\prime \prime}$, but this is impossible. So there is through $P$, and in a given plane containing $a$, not more than one perpendicular to $a$.

Theorem 9. If in the plane of two intersecting straight lines there is a perpendicular to one of them, then there is in this plane a perpendicular to the other one.

Proof. Suppose that the straight lines $a$ and $b$ intersect in a point $O$ and there is a perpendicular to $a$ lying in the plane $a b$. Then, if $B$ is any point of $b$ other than $O$, it follows, by Theorem 8, that there is a point $A$ on $a$ such that $B A$ is perpendicular to $a$. In case $A$ is $O$, then $a$ is perpendicular to $b$, and the conclusion of Theorem 9 is evidently verified. In case $A$ is not $O$, then there exist points $A^{\prime}$ and $B^{\prime}$, on $b$ and $a$ respectively, such that $O A \equiv O A^{\prime}$, 
$O B \equiv O B^{\prime} . \quad$ By Theorems 12 and $17, \Varangle B A O \equiv \Varangle B^{\prime} A^{\prime} O$. But $\Varangle B A O$ is a right angle. Hence, by the corollary to Definition $7, \Varangle B^{\prime} A^{\prime} O$ is a right angle. So $B A$ is perpendicular to $b$.

Theorem 10. If $a$ is any straight line and $P$ is any point, then in a given plane $\alpha$ containing $P$ and $a$ there is, through $P$, one and only one straight line perpendicular to $a$.

Proof. By Theorem 6, there exists in $\alpha$ some straight line $a^{\prime}$ to which there is a perpendicular lying in $\alpha$. 'In case $a^{\prime}$ is not $a$, suppose $A$ and $A^{\prime}$ are two points of $a$ and $a^{\prime}$ respectively. By hypothesis and Theorem 9 there is in $\alpha$ a perpendicular to $A A^{\prime}$, and therefore, by Theorem 9 , there is in $\alpha$ a perpendicular to $a$. Hence, by Theorem 8 , through each point of $\alpha$ there is, in $\alpha$, one and only one perpendicular to $a$.

Theorem 11. If $a$ and $b$ are two intersecting straight lines then there exist in the plane $a b$ straight lines $a^{\prime}$ and $b^{\prime}$ which are perpendicular to $a$ and $b$ respectively and are such that each angle that $a^{\prime}$ makes with $a \equiv$ each angle that $b^{\prime}$ makes with $b$.

Proof. See Theorem 10 and proof of Theorem 9.

\section{§ 4. Consequences of $O, C, A_{-2}$ and $A_{1}$.}

In $\S 2$ it was shown that $A_{1}$ as well as $A_{-2}$ was a consequence of $O, C$ and $M$. On the basis of $O, C, A_{1}$ and $A_{-2}$ we have the following propositions :

Theorem 1. . Any two coplanar right angles are congruent to each other.

Proof. If a side of one right angle is collinear with a side of another which is coplanar with it, it may be easily seen, with help of $A_{1}$, Definition 7 and Theorems 1, 15, 13 and 14, that they are congruent to each other. From this result and Theorem 11 of $\S 3$, it follows that two coplanar right angles are congruent to each other if the straight line wich contains a side of one intersects the straight line which contains a side of the other. From this, $O$, Theorem 14 of $\S 1$, and Theorem 10 of $\S 3$, it follows that any two coplanar right angles are congruent to each other.

Theorem 2*. If $A, B, C$ are not collinear and $D^{\prime}, A^{\prime}, B^{\prime}$ are not collinear, but $A, B, C, D^{\prime}, A^{\prime}, B^{\prime}$ are coplanar, then, in the half-plane $D^{\prime}-A^{\prime} B^{\prime}$, there is a point $C^{\prime}$ such that $\Varangle B A C \equiv \Varangle B^{\prime} A^{\prime} C^{\prime}$.

Proof. According to Theorem 10 of $\S 3$, there exist points, $E$ in the halfplane $C-A B$, and $E^{\prime}$ in the half-plane $D^{\prime}-A^{\prime} B^{\prime}$, such that $\Varangle E A B$ and $\Varangle E^{\prime} A^{\prime} B^{\prime}$ are right angles. According to Theorem 1, $\forall E A B \equiv E^{\prime} A^{\prime} B^{\prime}$. Suppose ray $A C$ lies within $\Varangle E A B$. Then, according to Theorem 18 of $\S 1$ and Theorem 1 of $\S 4$, there exists, in $\Varangle E^{\prime} A^{\prime} B^{\prime}$, a ray $A^{\prime} C^{\prime}$, such that $\Varangle B A C \equiv \Varangle B^{\prime} A^{\prime} C^{\prime}$. Proceed in a similar manner if $A C$ is within $\Varangle B_{1} A E$ (where $B_{1} A B$ ).

\footnotetext{
* Cl. HilberT's, IV, 4, loc. cit., page 14.
} 
Corollary. If $A B C$ and $A^{\prime} B^{\prime} C^{\prime}$ are two coplanar angles then either $\Varangle A B C<\Varangle A^{\prime} B^{\prime} C^{\prime}, \Varangle A B C \equiv \Varangle A^{\prime} B^{\prime} C^{\prime}$ or $\Varangle A B C>\Varangle A^{\prime} B^{\prime} C^{\prime}$.*

Theorem 3. If $A B C$ is a triangle and $B$ is between $A$ and $D$, then $\Varangle D B C>\Varangle C A B$.

Proof. By hypothesis and Theorem 3 of $\S 3$, neither $\Varangle D B C<\Varangle C A B$ nor $\Varangle D B C \equiv \Varangle C A B$. Hence, by corollary to Theorem 2 of $\S 4$, $\Varangle D B C>\Varangle C A B$.

Theorem 4. If $A B C$ is a triangle and $B C>A C$, then $\Varangle B A C>\Varangle A B C$ and, conversely, if $\Varangle B A C>\Varangle A B C$, then $B C>A C$.

Proof. I. Suppose $B C>A C$. Then, by Definition 1, there exists between $B$ and $C$ a point $D$ such that $A C \equiv C D$. Since $D$ is between $B$ and $C$, it easily follows, from $O$ and Definition 6, that $\Varangle B A C>\Varangle D A C$. But, by construction and Theorem 21 of $\S 1, \Varangle D A C \equiv \Varangle A D C$. Hence, by Theorems 12 and 19 of $\S 1, \Varangle B A C>\Varangle C D A$. But, by Theorem 3 of $\S 4$, $\Varangle C D A>\Varangle A B C$. Hence by Theorem 20 of $\S 1, \Varangle B A C>\Varangle A B C$.

2. Conversely, suppose $\Varangle B A C>\Varangle A B C$. By Theorem 8 of $\S 1$ either $B C<A C, B C \equiv A C$ or $B C>A C$. If $B C<A C$ or $B C \equiv A C$ then, by Theorem 21 of $\S 1$ and first part of present theorem, either $\Varangle B A C<\Varangle A B C$ or $\Varangle B A C \equiv \Varangle A B C$; and each of these, by Theorem 2 of $\S 3$, is contrary to hypothesis. Hence $B C>A C$.

Theorem 5. If $\Varangle A B C$ is a right angle, or $\Varangle A B C>$ a right angle, of the plane $A B C$, then $A C>A B$.

Proof. From hypotheses, Definitions 6 and 7, Theorems 19, 20 and 12 of $\S 1$, Theorems 3,2 and 10 of $\S 3$ and Theorem 1 of $\S 4$, it follows that neither $\Varangle A C B \equiv \Varangle A B C$ nor $\Varangle A C B>\Varangle A B C$. Hence, by the corollary to Theorem 2 of $\S 4, \Varangle A C B<\Varangle A B C$. Hence, by Theoren 4 of $\S 4$ and Definition $6, A C>A B$.

Theorem 6. If $A B C$ is a triangle, then $A B+A C>B C$.

Proof. By $C_{t_{a}}$, there exists a point $D$ such that $C A D$ and $A B \equiv A D$. By 'Theorem 21 of $\S 1 \Varangle B D C \equiv \Varangle D B A$. Hence, by $O$ and Definition 6, $\Varangle D B C>\Varangle C D B$, Hence, by Theorem 4 of $\S 4, D C>B C$. But, according to Definition 2, $D C=A B+B C$. Hence $A B+A C>B C$.

Corollary. If $A B C$ is a triangle and $A B>A C$, then $A B-A C<B C$.

\section{§ 5. Euclidean and Bolyai-Lobachevskian geometry.}

It is desired to prove that from $O, C, K$ and $P_{0}$ follows Euclidean geometry and from $O, C, K$ and the denial of $P_{0}$ follows Bolyai-Lobachevskian geometry.

Definition 8. Two straight lines are said to be parallel to each other if they lie in the same plane and have no point in common.

Theorems $1-5$ are based on the assumptions $O, C, K$.

* Cl. Theorem 2 of $\$ 3$. 
Theorem 1. Every segment has a middle point.

Proof. Suppose $A B$ is a segment. Certainly there exists a point $X$, of $A B$, such that $A X<X B$ and a point $Y$, of $A B$, such that $A Y>Y B$. For suppose $P$ is any point of $A B$. By Theorem 8 of $\S 1$, either $A B<P B$, $A P \equiv P B$ or $A P>P B$. If $A P>P B$, then, by Theorems 6 and 3 of $\S 1$, there is a point $P^{\prime}$ of $A B$ such that $A P \equiv B P^{\prime}$ and $P B \equiv P^{\prime} A$ and, by Theorem 7 of $\S 1$ and Definition 1 , it follows that $A P^{\prime}<P^{\prime} B$. Similarly, if $A P<P B$, there exists a point $P^{\prime}$ such that $A P^{\prime}>P B$. Finally, if $A P \equiv P B$, any point $D$ between $A$ and $P$ would be such that $A D<D P$, and there would exist a point $D^{\prime}$ such that $A D^{\prime}>D^{\prime} P$. It is true then that there is at least one point $X$ of $A B$ such that $A X<X B$ and at least one point $Y$ of $A B$ such that $A Y>Y B$. By Theorem 8 of $\S 1 Y$ could not be identical with $X$. The points of $A B$ may then be divided into two classes, the class, $[X]$, of all points. such that $A X<X B$ and the class, [Y], of all other points of $A B$. By Definition 1, $O$, and Theorems 9 and 8 of $\S 1$ it is clear that no $X$ is between two $Y$ 's and no $Y$ is between two $X$ 's. Hence, by $K$, there exists a point $M$ such that $X M Y$ for every $X$ and $Y$ which are different from $M$. Șuppose it were true that $A M<M B$. Then, by Theorems 6 and 3 of $\S 1$, there would exist a point $N$ of $A B$ such that $A M \equiv B N$ and $M B \equiv N A$. Clearly $M$ would be between $A$ and $N$. Hence, by a previous argument, it would follow that there exists a point $L$, between $M$ and $N$, such that $M L<L N$. But, from this together with the fact that $A M \equiv B N$, it would follow, from Definition 1 and Theorems 10 and 7 of $\S 1$, that $A L<L B$. But this would be impossible. For then $L$ would be an $X$ and we would thus have an $X$ such that $A M X$. Similarly it would be impossible that $A M>M B$. Hence $A M \equiv M B$.

This theorem having been established, it is clear that every proposition, given in the preceding part of this paper as a consequence of $O, C$ and $M$, is also a consequence of $O, C$ and $K$. Such propositions will therefore be freely referred to in this section. It is to be remembered that $A_{1}$ and $A_{-2}$ are propositions of this kind.

Definition 9. If $O$ is a point of a plane $\beta$ and $s$ is a segment, then the set of all points $[P]$ of the plane $\beta$, such that $O P \equiv s$ is called a circle.

Theorem 2. Every circle is a Jordan curve.

Proof. In his article "Theory of plane curves in non-metrical analysis situs,"* VEBLEN has given three sets of conditions as sufficient in order that a simple closed set of points, existing in a space in which $K$ and the plane axioms of the set $O$ hold true, should be a Jordan curve. These conditions are called the conditions of linear order, ordinal continuity, and geometrical continuity respectively.

* These Transaotions, vol. 6 (1905), lp. 83-98. 
If $O$ is the center of a circle $\alpha$, then every ray starting from $O$ contains one and only one point of $\alpha$.

The points $A, B, C, D$ of $\alpha$ are said to be in the order $A B C D$ if the rays $O A, O B, O C, O D$ are in the order $O A-O B-O C-O D$. From $O, K$ and this correspondence, it follows that the points of $\alpha$ satisfy, with reference to this order, the conditions of linear order and ordinal continuity.

The condition of geometrical continuity will evidently be satisfied if the proposition can be established that, given any point $P$ of $\alpha$ and any triangle $\Delta_{P}$ which contains $P$, then there exist in that triangle two points, $P_{1}$ and $P_{2}$ of $o$, such that every point on the are $P_{1} P P_{2}$ will lie within $\Delta_{P}$. To establish this proposition, proceed as follows : Given that $\Delta_{P}$ contains the point $P$ of the circle $\alpha$, consider a segment $s$ which is shorter than each of the perpendiculars from $O$ to the sides of $\Delta_{P}$. These perpendiculars and this segment exist by Theorem 10 of $\S 3$, Theorems 7,8 and 9 of $\S 1$, Definition 1 and $O$. By hypothesis, $O$, Theorem 5 of $\S 4$ and Detinition 1 and Theorem 9 of $\S 1$, it follows that every point $P^{\prime}$, such that $P P^{\prime}<s$, must be within $\Delta_{P}$. By Theorem 10 of $\S 3$, there exists, in the plane of $\alpha$, a straight line $a$ perpendicular to $O P$ at the point $P$. On $a$ there exist, by Theorem 1 of. $\S 1$, two points $E$ and $F$, one on each side of $P$, such that $s \equiv P E$ and $s \equiv P F$. Each of the rays $O E$ and $O F$ contains a point of $\alpha$. Call these points $P_{1}$ and $P_{2}$ respectively. Let $X$ be any point of $\alpha$ on the arc $P_{1} P P_{2}$. Then either $X$ is $P$ or it is in $\Varangle P O E$ or $\Varangle P O F$. Suppose $X$ is in $\Varangle P O E$. Then the ray $O X$ contains a point $M$ such that $P M E$. By Theorem 5 of $\S 4, O M>O P$. Hence $O M>O X$ and therefore $O X M$. But from the fact that $O P \equiv O X$ and Theorem 21 of $\S 1$ it follows that $\Varangle O X P \equiv \Varangle O P X$, and thus (see Theorem 3 of $\S 3) \Varangle O X P<$ a right angle (of the plane $\alpha$ ). Hence clearly, $\Varangle P X M>\mathbf{a}$ right angle (of the plane $\alpha$ ) and therefore, by Theorem 5 of $\S 4$, it follows that $P X<P M$. But $P M<P E$ and $P E \equiv s$. Hence $P X<s$ and therefore, by what has already been established, $X$ is within $\Delta_{P}$. This same conclusion would, of course, have been reached had $X$ been in $\Varangle F O P$. Hence, if $X$ is on the arc $P_{1} P P_{2}$, it lies within the triangle $\Delta_{P}$. It is true then that every circle is a Jordan curve.

Notation. $\mathfrak{A}_{r}$ means a circle whose center is $A$ and whose radius is congruent to $r$.

Theorem 3. Any point $P$ in the plane of the circle $D_{r}$ is within, on, or without $D_{r}$, according as $O P<r, O P \equiv r$, or $O P>r$.

Proof. If $P$ is any point in the plane of $D_{r}$ such that $O P>r$, then, with the help of Theorem 5 of $\S 4$, it may be, seen that the straight line which lies in the plane of $D_{r}$ and is perpendicular to $O P$ at the point $P$ has no point in common with $D_{r}$. Hence, by the theory of Jordan curves, it follows that $P$ is without $D_{r}$. With the help of $O$, Definition 7 and Theorem 5 of $\S 4$, it 
may be seen that all points $P$ such that $O P<r$ lie in one of the regions into which $D_{r}$ divides its plane. But it has already been established that all points such that $O P>r$ lie in the outside region, and moreover the two regions, into which $D_{r}$ divides all those points of its plane which do not lie on it, are nonvacuous and, finally, for every one of these points, either $O P<r$ or $O P>r$. Hence all points $P$ such that $O P<r$ must lie within $D_{r}$.

Theorem 4. If $A$ and $A^{\prime}$ are two points and $r$ and $r^{\prime}$ are two segments such that $r+r^{\prime}>A A^{\prime}$ but either $r=r^{\prime}$ or $r-r^{\prime}<A A^{\prime}$, then, if $\mathfrak{A}_{r}$ and $\mathfrak{A}_{r}^{\prime}$ are coplanar, they have two points in common, one on each side of $A A^{\prime}$.

Proof. $\mathfrak{A}_{r}$ has two points, $D$ and $E$, in common with the straight line $A A^{\prime}$. It follows, by hypothesis, Theorems of $\S 1$, Definition 9 and Theorem 2, that one of these points is within and the other is without $\mathfrak{A}_{r^{\prime}}^{\prime}$. But the points $D$ and $E$ are joined by two distinct segments of the Jordan curve $\mathfrak{A}_{r}$, one of these segments lying entirely on one side and the other one entirely on the opposite side of $D E$. Hence, since $\mathfrak{A}_{r^{\prime}}^{\prime}$ also is a Jordan curve, $\mathfrak{A}_{r}$ and $\mathfrak{A}_{r^{\prime}}^{\prime}$ intersect in two points, one on each side of $D E$.

Theorem 5.* If $A B C$ is any angle and $A^{\prime} B^{\prime}$ is any ray, then in any plane containing $A^{\prime} B^{\prime}$ there exists, on each side of the straight line $A^{\prime} B^{\prime}$, a point $C^{\prime}$ such that $\Varangle B A C \equiv \Varangle B^{\prime} A^{\prime} C^{\prime}$.

Proof. On ray $A^{\prime} B^{\prime}$ there exists, by Theorem 1 of $\S 1$, a point $B^{\prime \prime}$ such that $A B \equiv A^{\prime} B^{\prime}$. By hypothesis, Theorem 6 of $\S 4$ and its corollary, and Theorem 4 of $\S 5$, it follows that, on each side of $A^{\prime} B^{\prime}$, there is a point $C^{\prime \prime}$ such that $A C \equiv A^{\prime} C^{\prime \prime}$ and $B C \equiv B^{\prime} C^{\prime \prime}$. But $A B \equiv A^{\prime} B^{\prime \prime}$. Hence, by Definition $3, \Varangle B A C \equiv \Varangle B^{\prime} A^{\prime} C^{\prime}$.

If $P_{0}$ is assumed in addition to $O, C$, and $K$, then one has the following two theorems (6 and 7$)$.

Theorem 6. $\dagger$ If $b$ is any straight line of any plane $\beta$ then there is some point $B$, in the plane $\beta$ but not on $b$, such that through $B$ there is not more than one parallel to $b$.

Proof. According to $P_{0}$, there exists a straight line $b^{\prime}$ and a point $B^{\prime}$ such that through $B^{\prime}$ there is not more than one parallel to $b^{\prime}$. On $b^{\prime}$ there exist two points $C^{\prime}$ and $D^{\prime}$. If $b$ is any straight line in any plane $\beta$ then on $b$ there exist, by $O$ and $C_{1_{a}}$, two points $C$ and $D$ such that $C^{\prime} D^{\prime} \equiv C D$. Furthermore, by Theorem 5 of $\S 5$, there exists in the plane $\beta$ a point $B^{\prime \prime}$ such that $\Varangle D^{\prime} C^{\prime} B^{\prime} \equiv \Varangle D C B^{\prime \prime}$. By Theorem 1 of $\S 1$, there exists on ray $C B^{\prime \prime}$ a point $B$ such that $C^{\prime} B^{\prime} \equiv C B$. Suppose that through $B$ there are two parallels to $b$. Then one of these has a point $E$ and the other a point $F$ on that side of $B C$ on which $D$ lies. By Theorem 5 of $\S 5$, there exist in the plane $D^{\prime} C^{\prime} B^{\prime}$,

* See HiLberT's IV, 4.

† See VebLeN's XII. It may here be remarked that VhBLEN's form of statement laoks the proviso that $B$ should not lie on $b$.

Trans. Am. Math. Soc. 33 
and on that side of $B^{\prime} C^{\prime}$ on which $D^{\prime}$ lies, two points, $E^{\prime}$ and $F^{\prime}$, such that $\Varangle C B E \equiv \Varangle C^{\prime} B^{\prime} E^{\prime}$ and $\Varangle C B F \equiv \Varangle C^{\prime} B^{\prime} F^{\prime \prime}$. By Theorem 13 of $\S 1$, and $A_{-2}$, the straight line $B^{\prime} F^{\prime}$ must be distinct from the straight line $B^{\prime} E^{\prime}$. But neither of these straight lines can have a point in common with $C^{\prime} D^{\prime}$. For suppose the ray $B^{\prime} F^{\prime}$ and the straight line $C^{\prime} D^{\prime}$ had a point $G^{\prime}$ in common. $G^{\prime}$ would evidently lie on the ray $C^{\prime} D^{\prime}$. By Theorem 1 of $\S 1$, there would exist, on the ray $C D$, a point $G$ such that $C^{\prime} G^{\prime} \equiv C G$. Then one would have $C^{\prime} B^{\prime} \equiv C B, \Varangle G^{\prime} C^{\prime} B^{\prime} \equiv \Varangle G C B$ and $C^{\prime} G^{\prime} \equiv C G$. Hence, by Theorem 17 of $\S 1$ and Definition 5, it would be true that $\Varangle C^{\prime} B^{\prime} G^{\prime} \equiv \Varangle C B G$. But, by construction, $\Varangle C B F \equiv \Varangle C^{\prime} B^{\prime} G^{\prime}$. Hence, by Theorem 13 of $\S 1$ and $A_{-2}$ (which by Theorem 1 of $\S 2$ is a consequence of $O, C$, and $M$ ) the ray $B F$ would be the same as the ray $B G$. Hence ray $B F$ would have a point in common with ray $C G$, contrary to hypothesis. In a similar manner, with the help of Theorem 15 of $\S 1$, it could be shown that the other ray of the straight line $B^{\prime} F^{\prime}$ could not have a point in common with $C^{\prime} G^{\prime}$. Hence the straight lines $B^{\prime} F^{\prime}$ and $C^{\prime} D^{\prime}$ have no point in common. Similarly, $B^{\prime} E^{\prime}$ and $C^{\prime} D$ have no point in common. But then there exist, through $B^{\prime}$, two parallels to $b^{\prime}$, and this is contrary to hypothesis. Thus the assumption that through the point $B$ there are two parallels to $b$ would lead to an absurdity. Hence through $B$ there is not more than one parallel to $b$.

Theorem 7. If $a$ is any straight line and $A$ any point not on it, then through $A$ there is one and only one straight line parallel to $a$.

Proof. See Theorem 43 of Veblen's "A System of Axioms for Geometry."

In view of Theorems 1,4 and 6 of the present section, $C_{2}$, Theorems 1,3 , $5,12,14,17$ of $\S 1$ and Theorem 1 of $\S 2$, it is clear that from $O, C, K, P_{0}$ follows a geometry in which HILBERT's axioms of groups I-IV hold true. One may then proceed, as is, for example, indicated in HILBERT's Festschrift and HALSTED's Rational Geometry, to derive a theory of proportion, etc., and then, with the use of $K$, one may finally develop an analytic geometry exhibiting a one to one correspondence between the points of our geometry and the number triples of the real continuous number system, this correspondence being such as to preserve all relations of congruence and order. Thus would be established the following theorem :

Theorem 8. From $O, C, K$ and $P_{0}$, follows Euclidean geometry.

In case the contradictory of $P_{0}$ were assumed in addition to $O, C$ and $K$, then in place of Theorem 7 one would have Theorem 39 of Veblen's $A$ System of Axioms for Geometry and again a correspondence could be established between points and number triples, this correspondence also being such as to preserve all relations of order and congruence.* The following theorem would then be established :

* In this case congruence and order for this system of number triple would of course be introdnced by definitions different from those used in the case of the correspondence with Euclidean geometry. 
Theorem 9. From $O, C, K$ and the denial of $P_{0}$ follows Bolyai-Lobachevskian geometry.

It is thus seen that if $O, C$ and $K$ are true of a space, then that space must be either Euclidean or Bolyai-Lobachevskian.

\section{§6. Semi-quadratic geometry.}

In this section will be given several sets of assumptions, any one of which sets is sufficient for the establishment of semi-quadratic or plane semi-quadratic geometry. By semi-quadratic geometry is meant the set of propositions which follow from HILBERT's I-IV. In the case of such a geometry rigid motion is possible, there is a coördinate system and a theory of proportion ; and, speaking in terms of this theory of proportion, if $a_{1}, a_{2}, a_{3}, \cdots, a_{n}$ are any finite number of segments and $F\left(a_{1}, a_{2}, a_{3}, \cdots, a_{n}\right)$ is any rational function of these segments, then there exists a segment equal to $F\left(a_{1}, a_{2}, a_{3}, \ldots, a_{n}\right)$ and also a segment equal to $\sqrt{a^{2}+b^{2}}$.*

Use will be made of the following additional notations for propositions :

$R_{A}$ (equality of right angles). If $A B D, A^{\prime} B^{\prime} D^{\prime}, \Varangle A B C \doteq \Varangle C B D$ and $\Varangle A^{\prime} B^{\prime} C^{\prime} \equiv \Varangle C^{\prime} B^{\prime} D^{\prime}$, then $\Varangle A B C \equiv \Varangle A^{\prime} B^{\prime} C^{\prime}$.

By $R_{A}^{p l}$ is meant the proposition $R_{A}$ with the proviso that the angles $A B C$ and $A^{\prime} B^{\prime} C^{\prime}$ are coplanar. $\dagger$ This superscript, " $p l$," may sometimes be used in notations for other propositions in order that their application may be similarly restricted to the case of coplanar points.

$D_{a h}$ (shortest distance proposition). $\ddagger$ If $A, B$ and $C$ are non-collinear, $A C B^{\prime}$, $C B \equiv C B^{\prime}$, and $B^{\prime \prime}$ is a point on ray $A C$ such that $A B \equiv A B^{\prime \prime}$, then $A B^{\prime \prime} B^{\prime}$.

$P_{\text {or }}$ (perpendicular to a straight line). § If $A B C$, then in any plane containing these points there exists a point $D$ such that $\Varangle A B D \equiv \Varangle D B C$.

$A_{.} \|$If $A, B$ and $C$ are three non-collinear points and $A^{\prime}, B^{\prime}$ and $D^{\prime}$ are three non-collinear points and $B A \equiv B^{\prime} A^{\prime}$, then in the half-plane $D^{\prime} A^{\prime} B^{\prime}$ there exists one and only one point $C^{\prime}$ such that $A C \equiv A^{\prime} C^{\prime}$ and $B C \equiv B^{\prime} C^{\prime}$.

$A_{G}$ \%. If $\Varangle A B C$ and $\Varangle A^{\prime} B^{\prime} C^{\prime}$ are two angles and $B A \equiv B^{\prime} A^{\prime}$, then either there is a point $C^{\prime \prime}$ on the ray $B C$ such that $A^{\prime} C^{\prime} \equiv A C^{\prime \prime}$ and $B^{\prime} C^{\prime \prime} \equiv B C^{\prime \prime}$, or there is a point $C^{\prime \prime \prime}$ within $\Varangle A B C$ such that $A^{\prime} C^{\prime \prime} \equiv A C^{\prime \prime \prime}$ and $B^{\prime} C^{\prime} \equiv B C^{\prime \prime \prime}$, or there is a point $C^{\mathrm{iv}}$ within $\Varangle A^{\prime} B^{\prime} C^{\prime}$ such that $A C \equiv A^{\prime} C^{\mathrm{iv}}$ and $B C \equiv B^{\prime} C^{\mathrm{iv}}$; but for these given angles no two of these statements can both be true.

$P_{2}$. In every plane a there is a straight line a such that if $A$ is any point of

* See HilbrrT's Grundlagen der Geometrie.

† See Theorem 1 of $\$ 4$.

† See Theorem 6 of $\$ 4$.

§ For a stronger proposition see Theorem 10 of $\$ 3$.

$\|$ Cf. Theorem 2 of $\$ 4$ and $A_{-2}$ (see $\left.\$ 2\right)$. Cf. also HILBert's IV, 4.

II If $\Varangle A B C$ and $\Varangle A^{\prime} B^{\prime} C^{\nu}$ are two angles then eitber $\Varangle A^{\prime} B^{\prime} C^{\nu} \equiv \Varangle A B C$ or $\Varangle A^{\prime} B^{\prime} C^{\nu}>\Varangle A B C$ or $\Varangle A^{\prime} B^{\prime} C^{\prime}<\Varangle A B C$ but no two of these cases can ocour simultaneously. Cl. Definition 6, also Theorem 2 of $\$ 3$ and corollary to Theorem 2 of $\$ 4$. 
a not on a, then through $A$ and lying in a there is not more than one straight line which has no point in common with $a$.

Theorem 1. From $O^{p l}, C, M$, and $P_{2}$ follows plane semi-quadratic geometry.

Proof. See Theorems 1, 3, 5, 12, 14, 17 of $\S 1$ and Theorems 1 and 2 of $\S 2$ and Theorem 2 of $\S 4$. The proposition that through each point there is one and only one parallel to any given straight line may be proved with the help of $P_{2}$ by a method which is suggested in proof of Theorem 6 of $\S 5$.

Theorem 2. From $O^{p l}, C, A_{-2}, A_{1}, P_{2}$, follows plane semi-quadratic geometry.

Proof. In the proof above indicated for Theorem $1, M$ is used only to demonstrate $A_{-2}$ and $A_{1}$.

Lemma 1. From $O^{p l}, C$ and $D_{\text {sh }}$ follows $A_{-2}$.

Proof. Suppose $O, C$ and $D_{s h}$ are true and $A_{-2}$ is not. Then there exist three non-collinear points $A, B, C$, and a point $C^{\prime}$, all in the half-plane $C-A B$, such that $A C \equiv A C^{\prime \prime}$ and $B C \equiv B C^{\prime}$. According to $O$, there exist points $A^{\prime}, B^{\prime}$ such that $A C A^{\prime}, B C B^{\prime}$. There are five cases.

I. If $C^{\prime}$ is in $\Varangle A C B^{\prime}$, then segments $A C$ and $B C^{\prime}$ have a point $O$ in common. According to $D_{\text {sh }}, A O+O C^{\prime}>A C^{\prime}$ and $O C+B O>B C$. Thus $(A O+O C)+\left(O C^{\prime}+B O\right)>B C+A C^{\prime}$. Hence $A C+B C^{\prime}>B C+A C^{\prime}$; this is impossible in view of the hypothesis that $A C \equiv A C^{\prime}$ and $B C \equiv B C^{\prime}$.

II. If $C^{\prime}$ is in $\Varangle B C A^{\prime}$, argue in a similar manner.

III. If $C^{\prime}$ is in $\Varangle A C B$, then there exists a points $D$ such that $A C^{\prime} D$, $B D C$. Now $A C+C D>A D$. Hence $A C+C D+D B>A D+D B$, $A C+C B>A C^{\prime}+C^{\prime} D+D B$. But $C^{\prime} D+D B>C^{\prime} B$. Therefore $A C+C B>A C^{\prime}+C^{\prime} B$, and this is impossible in view of hypotheses.

IV. If $C^{\prime}$ is in $\Varangle A^{\prime} C B^{\prime}$, proceed as in case III.

$\mathrm{V}$. If $C^{\prime}$ is on the straight line $A C$ or the straight line $B C$, proceed with $C^{\prime}$ or $C^{\prime}$ as in case III with $D$.

Thus in any case it would be impossible that $O, C$ and $D_{s h}$ should be true and $A_{-2}$ false simultaneously.

Lemma 2. From $O, C$ and $P_{e r}$ it follows that through any point there is at least one perpendicular to any given straight line.

Proof. See the proof of Theorem 10 of $\S 3$.

Lemma 3. $A_{-2}$ is a consequence of $O, C$ and $P_{\text {ar }}$.

Proof. Suppose $C, A$ and $B$ are three non-collinear points and $C^{\prime}$ is a point in the half-plane $C-A B$ such that $C A \equiv C^{\prime} A$ and $C B \equiv C^{\prime} B$. By Lemma 2, there is, on the straight line $A B$, a point $D$ such that $C D$ is perpendicular to $A B$. In case $D$ coincides with $A$, then $\Varangle B A C$ is a right angle and therefore, by hypothesis and corollary to Definition 7 of $\S 3, \Varangle B A C$ is a right angle and hence, by $P_{\text {or }}$ and $C_{1_{b}}, C^{\prime \prime}$ is $C$. If $D$ does not coincide with $A$, then, by Theorem 17 of $\S 1, \Varangle A D C \equiv \Varangle A D C$ and $D C \equiv D C^{\prime}$. But 
$\Varangle A D C$ is a right angle. Hence, by the corollary to Definition 7 of $\S 3$, $\Varangle A D C^{\prime}$ is a right angle, and therefore, by $P_{\text {er }}$, ray $D C^{\prime}$ coincides with ray $D C$. But $D C \equiv D C^{\prime}$. Hence, by $C_{1_{b}}, C^{\prime}$ is $C$.

Theorem 3. From $O^{p l}, C, A_{1}, D_{a h}$ and $P_{2}$ follows plane semi-quadratic geometry.

Proof. See Lemma 1 and Theorem 2.

Theorem 4. From $O, C, A$, and $P_{2}$ follows semi-quadratic geometry.

Proof. Compare Theorems 1, 3, 5, 12, 14, 17 of $\S 1$ and Definition 3 and $A$, with HilberT's group IV of axioms. Use argument, concerning parallel proposition, suggested in proof of Theorem 1 of $\S 6$.

Theorem 5. From $O, C, A_{-2}, R_{A}$ and $P_{2}$ follows semi-quadratic geometry.

Proof. It may be seen, from the proof of Theorem 2 of $\S 4$, that $A_{\text {s }}$ is a consequence of $O, C, A_{-2}$ and $R_{A}$. But, by Theorem 4 of $\S 6$, from $O, C, A$. and $P_{2}$ follows semi-quadratic geometry.

Theorem 6. From $O, C, P_{e r}, R_{A}, P_{2}$ follows semi-quadratic geometry.

Proof. See Lemma 3 and Theorem 5.

Theorem 7. From $O, C, D_{s h}, R_{A}$ and $P_{2}$ follows semi-quadratic geometry.

Proof. See Lemma 1 and Theorem 5.

Theorem 8. From $O, C, A_{G}$ and $P_{2}$ follows semi-quadratic geometry.

Proof. According to Theorem 4, this theorem will be established if it is shown that $A$, is a consequence of $O, C$ and $A_{G}$.

Suppose $A, B$ and $C$ are three non-collinear points and $D^{\prime}, B^{\prime}$ and $E^{\prime}$ are three non-collinear points. On the ray $B^{\prime} D^{\prime}$ there is a point $A^{\prime}$ such that $B A \equiv B^{\prime} A^{\prime}$. To prove that in the half-plane $E^{\prime}-A^{\prime} B^{\prime}$ there is one and only one point $C^{\prime}$ such that $* B C \equiv B C^{\prime}$ and $A C \equiv A^{\prime} C^{\prime}$, argue as follows. According to $A_{G}$ either (I), there is a point $C^{\prime}$, on $B^{\prime} E^{\prime}$ or in $\Varangle A^{\prime} B^{\prime} E^{\prime}$, such that $B C \equiv B^{\prime} C^{\prime}$ and $A C \equiv A^{\prime} C^{\prime}$, or (II), there is a point $C^{\prime \prime}$ within $\Varangle A B C$, such that $\Varangle A B C^{\prime \prime} \equiv \Varangle A^{\prime} B^{\prime} E^{\prime}$. In this last case there is, on the ray $B C^{\prime \prime}$, a point $F$ such that $A F C$. By Theorem 11 of $\S 1$ there exists on ray $B^{\prime} E^{\prime}$ a point $F^{\prime}$ such that $A F \equiv A^{\prime} F^{\prime}$ and $B F \equiv B^{\prime} F^{\prime}$. By $C_{1_{a}}$ there is a point $C^{\prime}$ such that $A^{\prime} F^{\prime} C^{\prime}$ and $F C \equiv F^{\prime} C^{\prime}$. By $C_{3}$ and Theorem 11 of $\S 1, A C \equiv A^{\prime} C^{\prime}$ and $B C \equiv B^{\prime} C^{\prime}$.

Suppose there were, in the half-plane $E^{\prime}-A^{\prime} B^{\prime}$, two points $C^{\prime}$ and $C^{\prime \prime}$ such that $A C \equiv A^{\prime} C^{\prime}$ and $B C \equiv B^{\prime} C^{\prime \prime}$. Then, by $C_{1_{a}}$ and $O$, either $C^{\prime \prime}$ is within $\Varangle A^{\prime} B^{\prime} C^{\prime}$ or $C^{\prime}$ is within $\Varangle A^{\prime} B^{\prime} C^{\prime \prime}$. Suppose, for instance, $C^{\prime \prime}$ is within $\Varangle A^{\prime} B^{\prime} C^{\prime}$. Then, by hypothesis, Definition 3 and Theorems 11 and 18 of $\S 1$, there exists within $\Varangle A B C$ a point $C^{\prime \prime \prime}$ such that $B^{\prime} C^{\prime \prime} \equiv B C^{\prime \prime \prime}$ and $A^{\prime} C^{\prime \prime} \equiv A C^{\prime \prime \prime}$. But, by hypothesis, $C_{2}$ and Theorem 4 of $\S 1, A^{\prime} C^{\prime} \equiv A^{\prime} C^{\prime}$ and $B^{\prime} C^{\prime} \equiv B^{\prime} C^{\prime \prime}$. Hence, by $C_{2}, A^{\prime} C^{\prime} \equiv A C^{\prime \prime \prime}$ and $B^{\prime} C^{\prime} \equiv B C^{\prime \prime \prime}$. But $C^{\prime \prime}$ is within $\Varangle A^{\prime} B^{\prime} C^{\prime}$ and $A C \equiv A^{\prime} C^{\prime \prime}$ and $B C \equiv B^{\prime} C^{\prime \prime}$. Thus the

\footnotetext{
* Cr. A., page 503.
} 
supposition that there are two points $C^{\prime}$ and $C^{\prime \prime}$, in the half plane $E^{\prime} A^{\prime} B^{\prime}$ such that $A C \equiv A^{\prime} C^{\prime}, B C \equiv B^{\prime} C^{\prime}, A C \equiv A^{\prime} C^{\prime \prime}$ and $B C \equiv B^{\prime} C^{\prime \prime}$, would lead to a contradiction with $A_{G}$.

Theorem 7 is therefore established.

\section{§ 7. Geometry of the rule and compasses.}

In this paragraph will be considered several sets of assumptions, from any one of which sets follows a geometry in which all ordinary rule and compasses constructions are possible.

Two propositions concerning intersections of straight lines with circles, or of circles with each other, will be considered and will be referred to by means of the following notations:

$I_{1} \cdot{ }^{*}$ If $A, B$ and $C$ are non-collinear points and $P$ is between $A$ and $B$, then there is, on the straight line $D C$, a point $B^{\prime}$ such that $A B \equiv A B^{\prime}$.

$I_{2}+I_{f} A P D, P B P^{\prime}, A D^{\prime} P^{\prime}, P B \equiv B P^{\prime}, A D \equiv A D^{\prime}$, and all of these points lie in a plane, and this plane is decomposed by the straight line $P B$ into two regions, $\ddagger$ then, in each of these regions, there is a point $C$ such that $A C \equiv A D$ and $B C \equiv B P$.

Lemma 1. $M$ is a consequence of $O, C$, and $I_{2}$.

Proof. Suppose $A$ and $B$ are two different points. From $O$ and $C$ it easily follows that there is a point $P$, between $A$ and $B$, such that $A P>P B$. Then there is, by Theorems 3 and 6 of $\S 1$, a point $P^{\prime}$ such that $A P^{\prime} B$, $A P^{\prime} \equiv P B$, and $P^{\prime} B \equiv A P$. Evidently $A P^{\prime} P$. Hence, by $I_{2}$, there exist two points $D$ and $D^{\prime}$, on opposite sides of $A B$, such that

$$
B D \equiv A D \equiv B D^{\prime} \equiv A D^{\prime} \equiv A P \text {. }
$$

Segment $D D^{\prime}$ meets the straight line $A B$ in a point $M$. According to Definitions 3,5 and Theorems 3 and 17 of $\S 1, M A \equiv M B$. Thus ever $y$ segment has a middle point.

Lemmia 2. $I_{2}$ is a consequence of $O, C, M, P_{2}$ and $I_{1}$.

Proof. According to Theorem 1 of $\S 6$, plane semi-quadratic geometry is a consequence of $O^{p l}, C, M, P_{2}$. So a theory of proportion and an analytic geometry may be introduced. Now suppose one extremity of a diameter of $\mathfrak{E}_{r^{\prime}}^{\prime}$ is within $\mathfrak{C}_{r}$ and its other extremity is without $\mathfrak{C}_{r}$. Take $C C^{\prime}$ as $x$-axis and a perpendicular to $C C^{\prime}$ at the point $C$ as $y$-axis. Equations of $\mathbb{E}_{r}$ and $\mathfrak{C}_{r^{\prime}}^{\prime}$, referred to these axes, are $x^{2}+y^{2}=r^{2}$ and $(x-a)^{2}+y^{2}=r^{\prime 2}$ respectively, where $a$ is the abscissa of $C^{\prime}$. It is evident, in view of $I_{1}$, that for every value,

* If a straight line lies in the plane of a circle and has a point within that circle then it intersects the circle.

† If a semicircle has one extremity, $P$, within and the other, $P^{\prime}$, without a circle with which it is coplanar, then it bas a point in common with that circle.

$\ddagger$ Cf. V RBLEN, loc. cit., pages 363 and 364 . 
$x^{\prime}$, of $x$ between $-r$ and $+r$, there is a value, $y^{\prime}$, of $y$ such that $\left(x^{\prime}, y^{\prime}\right)$ satisfies $x^{2}+y^{2}=r^{2}$. With help of the fact that $r+r^{\prime}>a, a+r>r^{\prime}$ and $a+r^{\prime}>r$, it may be seen that $\frac{1}{2} a^{-1}\left(r^{2}+a^{2}-{r^{\prime}}^{2}\right)$ is between $-r$ and $+r$. Take for $x^{\prime}$ this value. Then

$$
y^{\prime}= \pm \sqrt{r^{\prime 2}-x^{\prime 2}}
$$

and, no matter which sign we take, these values of $x^{\prime}$ and $y^{\prime}$ evidently satisfy $(x-a)^{2}+y^{2}=r^{\prime 2}$ as well as $x^{2}+y^{2}=r^{2}$. Thus $\mathfrak{E}_{r}$ and $\mathfrak{S}_{.}^{\prime}$ intersect in two points, one on each side of $C C^{\prime}$.

Lemma, 3. $I_{1}$ is a consequence of $O, C$ and $I_{2}$.

Proof. From $\mathrm{O}, \mathrm{C}$ and $I_{2}, M$ follows, according to Lemma 1. Now suppose the straight line $p$ lies in the plane of the circle $\mathbb{E}_{r}$ and passes through a point $P$ which is within that circle. If $P$ is the same as $C$, then, by Theorem 1 of $\S 1, p$ evidently has a point in common with $\mathcal{E}_{r}$. If $P$ is different from $C$, it follows from $O, C$ and $M$ (see Theorem 10 of $\S 3$ ), that there exists on $p$ a point, $D$ such that $C D$ is perpendicular to $p$. By $C_{1_{a}}$ there is a point $C^{\prime}$ such that $C D C^{\prime}$ and $C D \equiv D C^{\prime}$. Then, evidently, according to hypothesis, Theorem 5 of $\S 4$, Theorems 1 and 2 of $\S 2$, and $I_{2}$, $\mathbb{E}_{r}$ and $\mathfrak{E}_{r}^{\prime}$ have a point $F$ in ccmmon. Hence, according to Definitions 3 and $7, F D$ is perpendicular to $C D$. Hence, by Theorem 10 of $\S 3, F$ lies on the straight line $p$. So $p$ and $\mathfrak{C}_{r}$ have a point $F$ in common.

Theorem 1. From $\mathrm{O}, \mathrm{C}, \mathrm{I}_{2}$, and $P_{2}$ follows a "Geometry of the Rule and Compasses."

Proof. With the help of Theorem 1 of $\S 6$ it may easily be seen that a geometry of the rule and compasses for each plane follows from $\mathrm{O}, \mathrm{C}, \mathrm{M}, \mathrm{P}_{2}$ and $\mathrm{I}_{2}$. Our present theorem will then be established if it is proved, (I), that $M$ follows from $O, C, P_{2}$ and $I_{2}$, and, (II), that a geometry of the rule and compasses follows from $I_{3}, P_{2}, O$, and geometry of the rule and compasses for each plane. Now $M$ follows from $O, C, P_{2}$ and $I_{2}$, according to Lemma 1 ; and (II) is evident in view of the fact that Theorem 5 of $\S 5$ is proved as a logical consequence of $I_{2}$ and plane semi-quadratic geometry for each plane.

§ 8. Independence of each assumption in the set composed of $K, P_{0}, C$ and $O$.

The following "independence examples" are constructed to show that each assumption of the set composed of $K, P_{0}, C$, and $O$ is independent of the remaining ones.

Example for order Axiom $I$. Use VeBlen's $K_{\mathrm{I}}$ (loc. cit., page 353).

Example for order Axiom III. Consider four points $A, B, C, D$ in the orders $A B C, A C B, A D B, B A D, B C A, B D C, C A D, C B D, C D A$, $D A C, D B A, D C B$. Consider that every segment $\equiv$ every segment.

Example for order Axiom $I V$. Use Vebles's $K_{\mathrm{IV}}$. Consider that every segment $\equiv$ every segment. 
Example for order Axiom VI. Points are all integers (including 0). $0 K 4$ and $4 K 0$ for every point $K$ except $-1,0,4$ and 5 . $-1,0$ and 4 are in the orders -104 and $40-1.0,4$ and 5 are in the orders 045 and 540 . If, in the ordinary sense, $0<A<4, C>4$ and $0<B<5$, then $\left\{\begin{array}{l}A B C \\ C B A\end{array}\right\}$ if and only if $B-A=C-B-1$. If, in the ordinary sense, $0<A<4, C<0$ and $-1<B<4$, then $\left\{\begin{array}{c}A B C \\ C B A\end{array}\right\}$ if and only if $A-B=B-C-1$. If $D$ and $E$ are two integers and there is no integer $C$ such that $D E C$ according to these definitions given above, then $\left\{\begin{array}{l}D E C \\ C E D\end{array}\right\}$ if and only if $E-C=D-E$. Every segment $\equiv$ every segment.

According to this plan one has the following orders :

$\begin{array}{llllllllllllll}8 & 4 & 1 & & 1 & 3 & 4 & -4 & 0 & 3 & & 3 & 0 & -4 \\ 7 & 4 & 2 & & 2 & 4 & 7 & -3 & 0 & 2 & & 2 & 0 & -3 \\ 6 & 4 & 3 & & 3 & 4 & 6 & -2 & 0 & 1 & & 1 & 0 & -2 \\ 6 & 3 & 1 & & 1 & 3 & 6 & -2 & 1 & 3 & & 3 & 1 & -2 \\ 5 & 4 & 0 & & 0 & 4 & 5 & -1 & 0 & 4 & & 4 & 0 & -1 \\ 5 & 3 & 2 & & 2 & 3 & 5 & -1 & 1 & 2 & & 2 & 1 & -1\end{array}$

$0 K 4$ and $4 K 0$ for every integer $K \neq-1,0,4$ or 5 ; and in case $A$ and $B$ are two integers which are not respectively the first and second element of any one of these triads, then $A B C$ if and only if $A-B=B-C$.

Example for order Axiom VII. VEBLEN's $K_{\mathrm{VII}}$. Every segment $\equiv$ every segment.

Example for order Axiom VIII. VebleN's $K_{\mathrm{VIII}}$. Every segment $\equiv$ every segment.

Example for order Axiom IX. VebLen's $K_{\mathrm{IX}}$. Congruence ordinary.

Example for order Axiom $X$. Veblen's $K_{10}$. Congruence ordinary.

Example for order Axiom $X I$ or $K$. Some non-Archimedean geometry, for example that of Hilbert (cf. Townsend's translation of HilberT's Grundlagen der Geometrie, page 34).

Example for $P_{0}$. Consider any of the ordinary proofs of the compatibility of Bolyai-Lobachevskian geornetry.

Example for $C_{1_{a}}$. Define points as the points of ordinary Euclidean space that lie on one side only of a given plane, consider these points to be ordered just as in the ordinary sense and two segments to be congruent to each other if and only if they are congruent in the ordinary sense. Then $K$ and $P_{0}$ are satisfied and so are all the assumptions of $C$ except $C_{\mathrm{t}_{\mathrm{a}}}$.

Example for $C_{1_{b}}$. Consider points to be all the points of ordinary Euclidean space ordered in the ordinary manner, but consider every segment as being congruent to itself and every other segment. Evidently $C_{1_{b}}$ is the only assumption of $K, P_{0}$ and $C$ that is not satisfied in this example.

Example for $C_{2}$. Consider points to be all the points of ordinary Euclidean 
space ordered in the usual manner, but consider that every segment is congruent to every segment which in the ordinary sense is just twice as long.

Example for $C_{3}$. Consider points to be the points of ordinary Euclidean space ordered in the usual manner, but consider that there is one segment $A B$ such that $C D \equiv C^{\prime} D^{\prime}$ if and only if $C^{\prime} D^{\prime}$ is congruent to $A B$ in the ordinary sense.

Example for $C_{4}$. Consider points to be the points of ordinary Euclidean space ordered in the usual manner. Consider a cerlain fixed plane $p$ and regard two segments neither of which is perpendicular to that plane as being congruent to each other if and only if their orthogonal projections upon that plane are congruent to each other in the ordinary sense $;$ in case only one of them is perpendicular to $p$, regard them as congruent if and only if this one is congruent in the ordinary sense to the projection of the other one upon $p$; finally, if they are both perpendicular to $p$, regard them as congruent if and only if they are congruent in the ordinary sense.

As was suggested by Professor E. H. Moore, the independence example here given for $C_{2}$ does not prove $C_{2}$ independent of $K, C$ and the negative of $P_{0}$. But all of the other independence examples here given (except, of course, that for $P_{0}$ ) do apply if the negative of $P_{0}$ is substituted for $P_{0}$ and Bolyai-Lobachevskian space is used instead of Euclidean. Of course the negative of $P_{0}$ is shown to be independent of $O, C$ and $K$ if point, order and congruence are taken as those of ordinary Euclidean space.

§ 9. Independence of each postulate in the set composed of $I_{3}, P_{2}, C$ and $O$.

Except for $C_{1_{a}}$ and $I_{2}$ use the same examples as in $\S 8$.

To prove $C_{1_{a}}$ independent, observe that all the assumptions $P_{0}, C, I_{2}$, except $C_{1_{a}}$, are satisfied, either " vacuously" or otherwise, if " points" are all the points of ordinary Euclidean space ordered as usual, no segment, however, being congruent to any segment. $C_{2}, C_{3}, C_{4}$ and $I_{2}$ are, in this case, "satisfied vacuously."

To prove $I_{2}$ independent, consider the space obtained by omitting all the points of Euclidean space except those whose coördinates are rationally expressible in terms of expressions of the form $\sqrt{r}$ where $r$ is an integer.

§ 10. Relation of parallel assumptions to introduction of congruence by definition.

With use of $O, K$ (or order "Axiom XI"), and Veblen's Axiom XII * (concerning parallels), congruence may $\dagger$ be introduced by definition, so that, if $O, K$ and XII are true of a space, then there must exist between the segments of that space a relation satisfying, for example, all of my assumptions $C$. But.

* See footnote $\dagger$ on page 501.

† VEBLEN, loo. cit., page 383. 
this is not true if XII is replaced by the weaker postulate $P_{0}$, as may be seen with help of the independence example obtained by considering a certain fixed plane in ordinary Euclidean space and regarding as "points" only those points of this space which are on a certain side of this fixed plane, these points being ordered as usual. Here $O, K\left(\right.$ also XI) and $P_{0}$ are satisfied. But there cannot exist among the segments of this space a relation satisfying assumptions $C$. For in that case this space would be, according to $\S 5$, ordinary Euclidean space and thus, in particular, through each point outside of any given line there would be only one parallel to that line, as is manifestly impossible.

\section{§11. Relation of continuity assumptions to introduction of congruence by definition.}

Theorem 1. It is not possible to prove that if the assumptions* $O, P_{\text {, }}$, $C_{1_{b}}, C_{2}, C_{3}, C_{4}$ (call this the set $S$ ), hold true of a space, then there exists a definition for the symbol " $\equiv "$ "such that the assumptions $C$ and $M$ will hold true with reference to the points and order in terms of which the assumptions of $S$ are stated.

Proof. Select any system $(O X, O Y, O Z)$ of three rectangular axes in ordinary Euclidean space and consider the space composed of all points whose coördinates with reference to this system are all rational, congruence and order relations being as usual. Call this space $U$ and let the terms "original order" and "original congruence" be understood here as meaning the order and congruence here indicated for this space. Clearly $O, P$, and all of the assumptions of $C$ except $C_{1_{a}}$ hold true of $U$ with reference to this order and congruence. Suppose a new meaning could be given to the congruence symbol " $\equiv$ " such that the assumptions $C$ and $M$ would hold true for the space $U$ with reference to the original order. Then, according to $\S 6$, all the theorems of plane semiquadratic geometry, in particular a theory of proportion, would hold true, with reference to this new congruence and the original order, for any planes in the space $U$. There would then exist on $O X$ a point $P$ such that, with reference to this theory of proportion, $O P \times O P=2 O I$ where $I$ is some point of $O X$ (e. g., such that $O I=$ original unit). $O P$ would then clearly not be, with reference to this new congruence, rational in terms of $O I$. But with use of $P_{\text {, it }}$ may be shown that any two segments which lie on $O X$, being rational in terms of each other with reference to the original congruence, would necessarily also be so with reference to this new congruence. To prove this let us first suppose that $A B$ and $C D$ are two segments of $O X$ and $A B \equiv C D$ according to original congruence. By $P$, there exists a parallelogram $A B E F$ in which $A B$ and $E F$

* By $P_{s}$ is meant the strong parallel assumption : If $a$ is any straight line and $A$ is any point not on $a$ then, in the plane $a A$, there is one and only one straight line which passes through $A$ and has no point in common with $a$. 
are opposite sides. Manifestly $E F C D$ will be a parallelogram in which $E F$ and $C D$ are opposite sides. But it is clear that, in terms of our new as well as in terms of our old congruence, the opposite side of a parallelogram must be songruent to each other. Therefore, in terms of this new congruence, $A B=E F$ and $E F \equiv C D$ and therefore $A B \equiv C D$. It is true then that if $A B$ and $C D$ are segments of $O X$ and $A B \equiv C D$ according to old congruence, then also $A B \equiv C D$ according to our new congruence. Now suppose that the segments $A B$ and $C D$, of the line $O X$, are given as rational in terms of one another (instead of simply congruent to one another) in terms of original congruence. Then there exists a segment $K L$, on $O X$, such that $A B$ can be divided into $m$ segments and $C D$ can be divided into $n$ segments, all congruent in the origipal sense, to $K L, m$ and $n$ being positive integers. But these $m+n$ segments of $O X$, being congruent to each other according to the original congruence, must also be congruent according to our new congruence, and thus $A B$ and $C D$ are rational in terms of one another according to new congruence. But it has been shown that, were such a new congruence possible, then there would exist on $O X$ two segments, $O P$ and $O I$, which would not be rational in terms of one another according to this congruence. A contradiction would thus be obtained and such a congruence would therefore be impossible.

\section{\$12. A question concerning the separation of the assumptions for a geometry into two sets.}

Theorem 1. It would be impossible to formulate a set (I) of assumptions expressed in terms of point and order alone and a set (II) of assumptions expressed in terms of point and congruence alone, such that any geometry satisfying the assumptions I and II must necessarily be ordinary Euclidean geometry with respect to the undefined symbols in terms of which I and II are stated.

Proof. Consider a system of three rectangular axes $O X, O Y, O Z$ in an ordinary Euclidean space $(E)$. Consider a paraboloid of revolution $(G)$ whose axis is $O Z$ and whose vertex is $O$. Let order be as usual but consider that segment $A B \equiv \operatorname{segment} A^{\prime} B^{\prime}$ if and only if

$$
\left(x_{A}-x_{B}\right)^{2}+\left(y_{A}-y_{B}\right)^{2}+\left(q_{A}-q_{B}\right)^{2}=\left(x_{A^{\prime}}^{i}-x_{B^{\prime}}\right)^{2}+\left(y_{A^{\prime}}-y_{B^{\prime}}\right)^{2}+\left(q_{A^{\prime}}-q_{B^{\prime}}\right)^{2} \text {, }
$$

the new coördinate, $q_{P}$, being defined as 0 if $P$ is on $G$, and otherwise as \pm the length of $S P$ where $S$ is the point in which $G$ is cut by a parallel to $O Z$ through the point $P$, and + or - is used according as $P$ is on the same side of the surface $G$ as is $Z$, or on the other side. Let $C^{\prime}$ designate this particular congruence. It may be seen that any statement concerning point and order alone that is true for Euclidean space must hold true for the space $E$ with reference to the congruence $C^{\prime}$. For if a straight line were defined as the locus of a point $P$ 
satisfying two linear equations of the first degree in $x_{P}, y_{P}, q_{P}$, and, in accordance with this, a new order $O^{\prime}$ were defined in the usual analytical manner, then one would have a Euclidean geometry concerning the points of the space $E$, the congruence $C^{\prime}$ and the order $O^{\prime}$. But also, of course, any statement concerning points and order alone which is true of ordinary Euclidean space is true of our original order for the particular space $E$. But manifestly space $E$ does not satisfy the theorems of ordinary Euclidean geometry with reference to ordinary order and the congruence $C^{\prime}$.

\section{$\S 13$. Certain queries.}

I do not know as yet whether $M^{*}$ is a consequence of $O \dagger$ and $C \dagger$. There are several other questions which could not be settled negatively without deciding this question. For example : Is $D_{s h} \ddagger$ a consequence of $O, C, A_{1} \S$ and $P_{2}$ ? $\ddagger$ Is $A_{1}$ a consequence of $O, C, D_{s h}$ and $P_{2}$ ? Is $R_{A} \ddagger$ a consequence of $O, C, A_{-2} \S$ and $P_{2}$ ? Is $A_{-2}$ a consequence of $O, C, R_{A}$ and $P_{2}$ ? Is $A_{1}$ a consequence of $O, C, A_{-2}$ and $P_{2}$ ? Is $A_{-2}$ a consequence of $O, C, A_{1}$ and $P_{2}$ ?

Another question is whether Theorem 1 of $\S 11$ would be true if $C_{1_{a}}$ were substituted for either $C_{\mathrm{1}_{b}}, C_{2}, C_{3}$ or $C_{4}$.

In $\S 6$ it was shown that from $O, C, M$ and $P_{2}$ follows a geometry for every plane of which all the theorems of plane semi-quadratic geometry hold true. Is then the semi-quadratic geometry of a three-space a consequence of $O, C, M$ and $P_{2}$ ? This could be answered in the affirmative if it could be shown that in every space for which $O, C$ and $M$ are true all right angles are congruent to each other. It can be proved $\|$ that in such a space all coplanur right angles are congruent to each other.

\footnotetext{
* See last sentence of \& 1 .

t See the opening pages of the paper.

$\ddagger$ See \& 6.

\& See 22.

I See Theorem 1 of $\& 4$.
} 